\title{
A Generalised Transmit and Receive Diversity Condition for Feedback Assisted MIMO Systems: Theory \& Applications in Full-Duplex Spatial Modulation
}

\author{
Rakshith Rajashekar, Member, IEEE, Marco Di Renzo, Senior Member, IEEE, K.V.S. Hari, Fellow, IEEE and L. \\ Hanzo, Fellow, IEEE
}

\begin{abstract}
It is widely exploited that the feedback assisted multiple-input multiple-output (MIMO) systems, which rely on channel state information (CSI) at the transmitter not only improve the spectral efficiency but also increase the attainable diversity gains. Owing to the limited bandwidth of the feedback channel, it is impractical to feed back perfect CSI or the transmit precoding (TPC) matrix to be used by the transmitter. This issue has been studied for over a decade now and it is addressed by feeding the TPC codeword index back to the transmitter. In this paper, we derive the conditions to be satisfied by the transmit and receive codebooks (TCBs and RCBs) for achieving full transmit and receive diversity gains. Furthermore, based on the conditions derived, we propose several $\mathrm{RCBs}$ by exploiting the properties of circulant matrices constructed with the aid of Cyclotomic polynomials. The proposed RCBs are shown to offer several benefits when employed in full-duplex (FD) spatial modulation (SM) systems, which include i) reduced hardware complexity of the self-interference (SI) cancellation circuitry ii) robustness to SI iii) maintain the diversity gain in the face of strong line-of-sight (LoS) channels. Furthermore, we study the performance of the proposed RCBs in an emerging drone communication scenario where several drones act as FD relays. Our simulation results show that the proposed RCBs indeed do attain the diversity gains predicted by our theoretical results. Specifically, in a dual-hop FD SM relay system the proposed receiver combiner selection (RCS) codebook is observed to give about $3 \mathrm{~dB}$ signal-to-noise (SNR) gain compared to the receive antenna selection (RAS) codebook without any substantial extra requirements. In the case of a triple-hop FD drone network, an SNR gain of about $2.5 \mathrm{~dB}$ is observed for our RCS codebook over a RAS codebook.
\end{abstract}

Index Terms-Joint transmit and receive processing, diversity gain, antenna selection, full-duplex, drones.

R. Rajashekar and L. Hanzo are with the School of ECS, University of Southampton, UK (e-mail: rmr1u14@soton.ac.uk, lh@ecs.soton.ac.uk).

K.V.S. Hari is with the Dept. of ECE, Indian Institute of Science, Bangalore, India (e-mail: hari@ece.iisc.ernet.in).

M. Di Renzo is with the Laboratoire des Signaux et Systèmes, CNRS, CentraleSupélec, Univ Paris Sud, Université Paris-Saclay, 3 rue Joliot Curie, Plateau du Moulon, 91192 Gif-sur-Yvette, France. (e-mail: marco.direnzo@12s.centralesupelec.fr). His work is supported in part by the Agence Nationale de la Recherche Scientifique (ANR) through the research project SpatialModulation (Société de l'Information et de la Communication - Action Plan 2015).

The financial support of the EPSRC projects EP/Noo4558/1 and EP/L018659/1, as well as of the European Research Council's Advanced Fellow Grant under the Beam-Me-Up project and of the Royal Society's Wolfson Research Merit Award is gratefully acknowledged.

\section{INTRODUCTION}

Multi antenna aided systems offer significantly higher system capacity than single-input single-output (SISO) systems [1], [2]. Apart from offering a higher spectral efficiency, the multiple-input multiple-output (MIMO) systems are also capable of providing diversity gains [3], which is indeed imperative for achieving a high integrity. Numerous techniques were conceived in the literature for attaining both transmit and receive diversity gains. These techniques can be broadly grouped into two categories, namely (i) open loop, and (ii) closed loop arrangements. The open loop techniques are predominantly represented by space-time block coding (STBC) [4], where the information bits to be transmitted are encoded into complexvalued space-time (ST) matrices, which are in turn transmitted through multiple transmit antennas. For instance, the $(2 \times 2)$ element Alamouti STBC [5] requires two active transmit antennas and two channel uses for its transmission, whereas a $\left(2^{l} \times 2^{l}\right)$-element orthogonal STBC [6] requires $2^{l}$ active transmit antennas and channel uses. Although, the orthogonal STBCs have the benefit of single-symbol maximum likelihood (ML) decodability, their transmission rate expressed in terms of encoded complex symbols per channel use reduces, as the number of transmit antennas (TA) is increased. Several STBCs were conceived in the literature that offer high diversity as well as multiplexing gains, which include the STBCs constructed from division algebras [7], the golden code [8], the perfect STBCs [9] etc. Regretfully, these STBCs impose excessive ML decoding complexity at the receiver. Another technique of attaining both transmit as well as receive diversity gains is to exploit the channel state information (CSI) both at the transmitter as well as at the receiver using closed loop techniques, where transmit precoding (TPC) and receiver combining matrices are employed. The optimal TPC and receiver combining matrices for a given channel realization are known to be the left and right singular matrices, respectively [3]. Since the bandwidth of the feedback channel is limited, it is not feasible to convey the full CSI or the right singular vectors of the channel to the transmitter. Thus, a limited feedback $(L F)$ approach is adopted, where a codeword is selected from a pre-determined codebook that maximizes a certain metric of interest. Some of the codebooks conceived for LF schemes include the popular Grassmannian codebook [10], unitary 
TPC-aided orthogonal STBCs [11], combined-beamforming and STBC-aided quantized feedback [12], algebraic rotation and threaded space-time architecture assisted codebooks [13] etc.

While MIMO systems offer the benefit of both diversity and multiplexing gains, they suffer from a high power consumption owing to power-hungry radio frequency (RF) chains. Spatial modulation (SM) [14]-[19] is a relatively new scheme conceived for overcoming the aforementioned drawback by employing only a single RF chain at the transmitter. Specifically, the SM system employs several TAs, but activates only a single TA for information transmission in each channel use. The specific choice of the activated TA depends on the information bits to be transmitted. Thus, the SM system implicitly conveys information through the active TA index as well as through the conventional symbol chosen from a classic signal set, such as QAM/PSK. An important benefit of the SM system is that it completely avoids inter-antenna interference at the receiver owing to using a single active TA and hence it is suitable for low-complexity ML detection [18]. However, SM is by no means flawless, since it suffers from the lack of transmit diversity gain owing to the single active TA. In order to overcome this drawback, several open-loop and closed-loop techniques were conceived. Analogous to the conventional open-loop MIMO system, several ST coding schemes were conceived [20]-[23] for improving the transmit diversity gain. Specifically, an Alamouti code aided SM arrangement was conceived in [20], while a complex interleaved orthogonal design was advocated in [21]. As a further development, a SM scheme employing Alamouti STBC having a cyclic structure was conceived in [22], while a SM scheme also relying on Alamouti STBC with phasors was conceived in [23]. All the aforementioned schemes achieve a transmit diversity order of two, while requiring two transmit RF chains, except for the scheme in [21], which requires a single transmit RF chain. Closed-loop techniques were also conceived for enhancing the integrity of SM systems [24]-[29]. Specifically, link-adaptive modulation was studied in [24], while both capacity based and Euclidean distance based antenna selection (EDAS) schemes were proposed in [25]. Furthermore, low-complexity antenna selection algorithms were proposed in [26], [27]. The transmit diversity order of EDAS was quantified in [28], while Sun et. al. [29] proposed a cross-entropy based method for reducing the search complexity of EDAS. In [30], Yang et. al. proposed an improved low-complexity implementation of EDAS by striking a beneficial performance vs. complexity trade-off. Recently, Sun et. al. [31] have proposed a reduced-dimensional EDAS-equivalent criterion, which results in the same performance as that of EDAS, albeit at a reduced complexity.

Against this background, the following are the contributions of this paper:

1) The existing literature on feedback-assisted SM schemes is mainly focused on attaining transmit diversity gain with the aid of TA subset selection, where the receiver is usually assumed to have multiple RF chains. Since employing fewer RF chains than the number of receive antennas can result in a significant power saving at the receiver, it is beneficial to consider joint transmit and receive antenna subset selection. In this regard, we first derive a generalized transmit and receive diversity condition for feedback-assisted MIMO systems. Based on this, we obtain a condition on the transmit and the receive codebooks that facilitate attaining full diversity despite using antenna subset selection ${ }^{1}$. Furthermore, we lay the foundations of dual-function feedback-assisted MIMO schemes, which combine STBC, SM, as well as antenna subset selection in a single joint designframework. This design-framework not only helps in viewing various modulation and coding schemes under a single umbrella, but also opens up a new avenue for designing them.

2) While MIMO systems significantly increase the spectral efficiency, it was shown that further throughput improvement is possible with the aid of full-duplex (FD) communication [35]-[37]. Owing to the benefit of having a nearly doubled throughput, FD communication has recently been studied also in the context of SM systems [38]-[40]. Specifically, Jiao et. al. [38] studied the attainable mutual information of a $(2 \times 2)$ FD SM system, while Raviteja et. al. [39] studied the performance of a dual-hop FD relay system employing SM. In [40], Zhang et. al. considered a FD two-way relay system employing SM and studied the attainable performance. Note that both the relay systems of [39] and [40] consider multiple receive antennas, which require both multiple RF chains as well as complex self-interference (SI) cancellation circuitry. With the aid of the results in 1), we propose an energy efficient antenna subset selection aided FD SM relay system, which achieves the full transmit and receive diversity gain, despite reducing the hardware requirements of the FD SM systems in [39], [40].

3) Lastly, as a practical application of the proposed FD SM relay system we consider multi-hop FD relaying in a drone-aided communication scenario [41]-[45], where multiple drones act as relays. Specifically, we consider the ill effects of the strong line-of-sight (LOS) channel of multi-hop drone relays, and propose methods of alleviating them with the aid of the results in 1) without compromising the attainable diversity gains.

The remainder of the paper is organized as follows. The generalized transmit and receive diversity condition for feedback assisted MIMO systems is derived in Section II and several examples are discussed. The proposed receiver processing schemes are presented in Section III. Our simulation results and discussions are discussed in Section IV, while Section V concludes the paper.

\footnotetext{
${ }^{1}$ Note that the problem of attaining both transmit as well as receive diversity gains has been extensively studied in the literature [32]-[34]. Specifically, the authors in [32] studied an Alamouti code [5] based transmission scheme that employs transmit as well as receive antenna selection. In [33], the authors propose receive antenna selection algorithms that maximize the system capacity. In [34], the authors study the performance of joint transmit and receive antenna selection algorithms in Nakagami fading channels. In contrast to the existing schemes, which essentially rely on antenna selection, the proposed approach provides a generalized framework for transmit and receive codebook selection, which essentially subsumes the antenna selection schemes as special cases.
} 
Notations: The notations of $(\cdot)^{H}$ and $(\cdot)^{T}$ indicate the Hermitian transpose and transpose of a vector/matrix, respectively, while $|\cdot|$ represents the magnitude of a complex quantity, or the cardinality of a given set. The uppercase boldface letters represent matrices and lowercase boldface letters represent vectors. The notations of $\|\cdot\|_{F}$ and $\|\cdot\|$ represent the Frobenious norm of a matrix and the two-norm of a vector, respectively. $\mathbf{H}([c: d],:)$ represents a matrix with rows $c, c+1, \ldots, d-1, d$ of $\mathbf{H}$ and $\mathbf{H}(:,[c: d])$ is a matrix with columns $c, c+1, \ldots, d-1, d$ of $\mathbf{H}$. The Kronecker product of the matrices $\mathbf{A}$ and $\mathbf{B}$ is represented by $\mathbf{A} \otimes \mathbf{B} \cdot \operatorname{span}(\mathbf{A})$ represents the space spanned by the columns of $\mathbf{A} . \operatorname{Tr}(\cdot)$ represents the trace of a matrix. Expected value of a random variable $Y$ is denoted by $\mathrm{E}(Y)$, while the smallest non-zero Eigenvalue of matrix $\mathbf{Y}$ is denoted by $\lambda_{s m}(\mathbf{Y})$. Furthermore, $Q(x)$ represents the tail probability of standard normal distribution given by $\frac{1}{\sqrt{2 \pi}} \int_{x}^{\infty} \exp \left(-\frac{u^{2}}{2}\right) d u$. A circularly symmetric complex-valued Gaussian distribution with mean $\mu$ and variance $\sigma^{2}$ is represented by $\mathcal{C N}\left(\mu, \sigma^{2}\right)$. $\mathbb{C}$ and $\mathbb{R}$ represent the field of complex and real numbers, respectively. $\boldsymbol{\Phi}_{l}(x)$ represents the $l^{\text {th }}$ Cyclotimc polynomial in $x$.

\section{Generalized Transmit and Receive Diversity Condition Proposed for FeEdback Assisted MIMO SYSTEMS}

\section{A. System Model}

Consider a MIMO system having $N_{r}$ receive and $N_{t}$ TAs. Let the number of RF chains at the transmitter be $M_{t} \leq N_{t}$. Let $\mathbf{X} \in \mathbb{C}^{N_{t} \times T}$ represent the transmit ST matrix, which requires $T$ channel uses. Let $\mathbf{Z} \in \mathbb{C}^{N_{r} \times N_{r}}$ represent the effective analog-to-baseband processing matrix ${ }^{2}$ at the receiver, which is chosen from a receive codebook $(\mathrm{RCB}) \mathcal{Z}$. Then, the received ST matrix is given by

$$
\mathbf{Y}=\sqrt{\frac{\rho}{M_{t}}} \mathbf{Z H X}+\mathbf{N} \quad \in \mathbb{C}^{N_{r} \times T},
$$

where $\rho$ represents the average signal-to-noise ratio (SNR) at each receive antenna, $\mathbf{H} \in \mathbb{C}^{N_{r} \times N_{t}}$ represents the channel matrix and $\mathbf{N} \in \mathbb{C}^{N_{r} \times T}$ represents the noise matrix, both of whose entries are from $\mathcal{C N}(0,1)$. If $\mathbf{X}$ belongs to a transmit codebook (TCB) $\mathcal{X}$, then the ML solution assuming perfect $\mathrm{CSI}$ at the receiver is given by

$$
\hat{\mathbf{X}}=\arg \min _{\mathbf{X} \in \mathcal{X}}\left\|\mathbf{Y}-\sqrt{\frac{\rho}{M_{t}}} \mathbf{Z H X}\right\|_{F}^{2} .
$$

\footnotetext{
${ }^{2}$ Please note that the receive processing matrix $\mathbf{Z}$ considered in the paper is the analog-to-baseband processing matrix, which is slightly different from the baseband post-prossing matrix generally considered in the literature, where the noise statistics change significantly, depending on $\mathbf{Z}$. The analog-to-baseband processing matrix models the analog domain processing at the receiver, such as the receive antenna selection, analog phase shift operation etc. which do not alter the noise statistics. Note that any change in the noise statistics due to receive processing is dealt with in Section III-B. Note that for the sake of presentation, we have considered $\mathbf{Z}$ to be orthonormal and hence the associated noise to be white. All the results in the paper hold even when $\mathbf{Z}$ is not orthonormal. In that case, $\mathbf{Z}$ is pre-multiplied by $\left(\mathbf{Z Z}^{H}\right)^{-1 / 2}$ in order to whiten the noise.
}

The attainable transmission rate of this scheme is given by

$$
R=\frac{\log _{2}(|\mathcal{X}|)}{T} \text { bpcu, }
$$

where bpcu stands for bits per channel use.

In case of feedback-assisted MIMO systems, the TCB $\mathcal{X}$ and the receive preprocessing matrix $\mathbf{Z}$ can be jointly selected as follows:

$$
(\mathbf{Z}, \mathcal{X}) \equiv\left(\mathbf{Z}_{j^{*}}, \mathcal{X}_{i^{*}}\right)=\arg \max _{\mathbf{Z}_{j} \in \mathcal{Z}, \mathcal{X}_{i} \in \overline{\mathcal{X}}} \mathrm{f}\left(\mathbf{H}, \mathbf{Z}_{j}, \mathcal{X}_{i}\right),
$$

where $\overline{\mathcal{X}}=\left\{\mathcal{X}_{1}, \mathcal{X}_{2}, \ldots, \mathcal{X}_{n}\right\}$ is a collection of TCBs, $\mathrm{f}(\cdot)$ can be any metric of interest such as the capacity [25], the minimum Euclidean distance [28] etc. Upon obtaining $\mathcal{X}=\mathcal{X}_{i^{*}}$ from (4), the receiver feeds the codebook index $i^{*}$ back to the transmitter, and uses the receive processing matrix $\mathbf{Z}=\mathbf{Z}_{j^{*}}$ for its subsequent reception. Upon receiving the optimal codebook index $i^{*}$, the transmitter uses $\mathcal{X}_{i^{*}}$ for subsequent data transmissions. In the next part of the paper, we derive the attainable diversity gain of the aforementioned feedback-assisted MIMO system by considering $f(\cdot)$ to be the minimum Euclidean distance (ED) between the receive constellation matrices. ${ }^{3}$

\section{B. Proposed Generalized Transmit and Receive Diversity Condition}

Let $\Delta \mathcal{X}_{i}=\left\{\mathbf{X}_{1}-\mathbf{X}_{2} \mid \mathbf{X}_{1}, \mathbf{X}_{2} \in \mathcal{X}_{i}, \mathbf{X}_{1} \neq \mathbf{X}_{2}\right\}$ be the set of difference matrices corresponding to the codebook $\mathcal{X}_{i}$ and

$\Delta \overline{\mathcal{X}}=\left\{\left[\mathbf{X}_{1}, \ldots, \mathbf{X}_{n}\right] \mid \mathbf{X}_{1} \in \Delta \mathcal{X}_{1}, \mathbf{X}_{2} \in \Delta \mathcal{X}_{2}, \ldots, \mathbf{X}_{n} \in \Delta \mathcal{X}_{n}\right\}$.

Let the feedback function be given by

$$
\mathbf{f}\left(\mathbf{H}, \mathbf{Z}_{j}, \mathcal{X}_{i}\right)=\min _{\mathbf{X} \in \Delta \mathcal{X}_{i}}\left\|\mathbf{Z}_{j} \mathbf{H X}\right\|_{F}^{2}
$$

which represents the minimum ED between the receive constellation matrices. Let $\mathbf{X}_{\min }(j, i)=$ $\arg \min _{\mathbf{X} \in \Delta \mathcal{X}_{i}}\left\|\mathbf{Z}_{j} \mathbf{H X X}\right\|^{2}$ for $1 \leq i \leq n, 1 \leq j \leq m$ and $\overline{\mathbf{X}}_{j}=\left[\mathbf{X}_{\min }(j, 1), \mathbf{X}_{\min }(j, 2), \ldots, \mathbf{X}_{\min }(j, n)\right]$.

Proposition 1: The feedback-assisted MIMO system employing (4) with $\mathrm{f}(\cdot)$ given in (6) attains a total diversity gain of $r=\operatorname{rank}\left(\sum_{j=1}^{m} \mathbf{B}_{j}\right)$, where $\mathbf{B}_{j}=\left(\mathbf{Z}_{j}^{H} \mathbf{Z}_{j}\right)^{T} \otimes \overline{\mathbf{X}}_{j} \overline{\mathbf{X}}_{j}^{H}$.

Proof: Please refer to Appendix A.

Let the RCB be represented by $\mathcal{Z}=\left\{\mathbf{Z}_{1}, \mathbf{Z}_{2}, \ldots, \mathbf{Z}_{m}\right\}$ and $\overline{\mathbf{Z}}$ represent the stacked $\mathrm{RCB}$ entries given by $\overline{\mathbf{Z}}=$ $\left[\mathbf{Z}_{1}^{T}, \mathbf{Z}_{2}^{T}, \ldots, \mathbf{Z}_{m}^{T}\right]^{T} \in \mathbb{C}^{m N_{r} \times N_{r}}$. The following proposition gives a necessary and sufficient condition to be satisfied by the RCB as well as by the TCBs for attaining full diversity order.

Proposition 2: A necessary and sufficient condition for attaining the full diversity order of $N_{t} N_{r}$ by the feedback assisted MIMO system employing (4) with $\mathrm{f}(\cdot)$ given in (6) is that $\operatorname{rank}(\overline{\mathbf{Z}})=N_{r}$ and $\min \{\operatorname{rank}(\overline{\mathbf{X}}) \mid \overline{\mathbf{X}} \in \Delta \overline{\mathcal{X}}\}=N_{t}$.

Proof: Please refer to Appendix B.

\footnotetext{
${ }^{3}$ Note that the feedback function given by (4) mainly depends on the channel realization $\mathbf{H}$ and the quality of the channel estimate available at the receiver depends on the amount of self-interference, which in turn affects the feedback function. We do not dwell on imperfect channel information scenario in this paper as it requires an explicit analysis, which is outside the scope of this work.
} 
Proposition 2 motivates us to consider only full-rank $\overline{\mathbf{Z}}$ in order to attain full receive diversity gain. The following corollary quantifies the minimum required number of codebook entries in RCB for attaining full receive diversity gain.

Corollary 1: If $\operatorname{rank}\left(\mathbf{Z}_{j}\right)=k$ for $1 \leq j \leq m$ and the rows of $\mathbf{Z}_{j_{1}}$ for $1 \leq j_{1} \leq m$ do not belong to the space spanned by the rows of $\left[\mathbf{Z}_{1}^{T} \cdots \mathbf{Z}_{k-1}^{T}, \mathbf{Z}_{k+1}^{T} \cdots \mathbf{Z}_{m}^{T}\right]^{T}$ where $k=j_{1}$, then the number of codebook entries $m$ in $\mathcal{Z}$ required for attaining full receive diversity order is $N_{r} / k$.

Proof: From Proposition 2, we have the requirement that $\operatorname{rank}(\overline{\mathbf{Z}})=N_{r}$ is maintained for attaining full receive diversity gain. If the rows of $\mathbf{Z}_{j_{1}}$ do not belong to the space spanned by the rows of $\left[\mathbf{Z}_{1}^{T} \cdots \mathbf{Z}_{k-1}^{T}, \mathbf{Z}_{k+1}^{T} \cdots \mathbf{Z}_{m}^{T}\right]^{T}$ where $k=j_{1}$ and $\operatorname{rank}\left(\mathbf{Z}_{j}\right)=k$, then we have $\operatorname{rank}(\overline{\mathbf{Z}})=k m$. If $\operatorname{rank}(\overline{\mathbf{Z}})=N_{r}$, then we have $m k=N_{r}$ and $m=N_{r} / k$. This concludes the proof.

The following examples provide TCB constructions along with their attainable diversity gains. The RCB constructions are presented in Section III in conjunction with their applications in FD systems.

Example 1: Let $N_{t}=8, T=1$ and

$\mathbf{m}_{1}=\left[\begin{array}{l}s \\ 0 \\ 0 \\ 0\end{array}\right], \mathbf{m}_{2}=\left[\begin{array}{l}0 \\ s \\ 0 \\ 0\end{array}\right], \mathbf{m}_{3}=\left[\begin{array}{l}0 \\ 0 \\ s \\ 0\end{array}\right], \mathbf{m}_{4}=\left[\begin{array}{l}0 \\ 0 \\ 0 \\ s\end{array}\right]$,

represent the SM vectors [14], where $s$ is from a QAM/PSK signal set. Furthermore, let the collection of TCBs be $\overline{\mathcal{X}}=$ $\left\{\mathcal{X}_{1}, \mathcal{X}_{2}\right\}$, where we have

$$
\mathcal{X}_{1}=\left\{\left[\begin{array}{c}
\mathbf{m}_{1} \\
\mathbf{o}
\end{array}\right],\left[\begin{array}{c}
\mathbf{m}_{2} \\
\mathbf{o}
\end{array}\right],\left[\begin{array}{c}
\mathbf{m}_{3} \\
\mathbf{o}
\end{array}\right],\left[\begin{array}{c}
\mathbf{m}_{4} \\
\mathbf{o}
\end{array}\right]\right\}
$$

and

$$
\mathcal{X}_{2}=\left\{\left[\begin{array}{c}
\mathbf{o} \\
\mathbf{m}_{1}
\end{array}\right],\left[\begin{array}{c}
\mathbf{o} \\
\mathbf{m}_{2}
\end{array}\right],\left[\begin{array}{c}
\mathbf{o} \\
\mathbf{m}_{3}
\end{array}\right],\left[\begin{array}{c}
\mathbf{o} \\
\mathbf{m}_{4}
\end{array}\right]\right\} .
$$

The vector o represents the all-zero vector of dimension $4 \times 1$. It is straightforward to see that $\min \{\operatorname{rank}(\overline{\mathbf{X}}) \mid \overline{\mathbf{X}} \in \Delta \overline{\mathcal{X}}\}=2$ and hence this scheme achieves a transmit diversity order 2.

Example 2: Let $N_{t}=8, T=2$ and

$$
\begin{gathered}
\mathbf{M}_{1}=\left[\begin{array}{cc}
\tilde{s}_{1} & 0 \\
0 & \tilde{s}_{2} \\
0 & 0 \\
0 & 0
\end{array}\right], \mathbf{M}_{2}=\left[\begin{array}{cc}
0 & 0 \\
\tilde{s}_{1} & 0 \\
0 & \tilde{s}_{2} \\
0 & 0
\end{array}\right], \\
\mathbf{M}_{3}=\left[\begin{array}{cc}
0 & 0 \\
0 & 0 \\
\tilde{s}_{1} & 0 \\
0 & \tilde{s}_{2}
\end{array}\right], \text { and } \mathbf{M}_{4}=\left[\begin{array}{cc}
0 & \tilde{s}_{2} \\
0 & 0 \\
0 & 0 \\
\tilde{s}_{1} & 0
\end{array}\right],
\end{gathered}
$$

represent the matrices from complex interleaved orthogonal design (CIOD) based SM [21], where $\tilde{s}_{1}$ and $\tilde{s}_{2}$ are coordinate interleaved symbols drawn from QAM/PSK signal sets. Furthermore, let the collection of TCBs be $\overline{\mathcal{X}}=\left\{\mathcal{X}_{1}, \mathcal{X}_{2}\right\}$, where we have

$$
\mathcal{X}_{1}=\left\{\left[\begin{array}{c}
\mathbf{M}_{1} \\
\mathbf{O}
\end{array}\right],\left[\begin{array}{c}
\mathbf{M}_{2} \\
\mathbf{O}
\end{array}\right],\left[\begin{array}{c}
\mathbf{M}_{3} \\
\mathbf{O}
\end{array}\right],\left[\begin{array}{c}
\mathbf{M}_{4} \\
\mathbf{O}
\end{array}\right]\right\}
$$

and

$$
\mathcal{X}_{2}=\left\{\left[\begin{array}{c}
\mathbf{O} \\
\mathbf{M}_{1}
\end{array}\right],\left[\begin{array}{c}
\mathbf{O} \\
\mathbf{M}_{2}
\end{array}\right],\left[\begin{array}{c}
\mathbf{O} \\
\mathbf{M}_{3}
\end{array}\right],\left[\begin{array}{c}
\mathbf{O} \\
\mathbf{M}_{4}
\end{array}\right]\right\} .
$$

The matrix $\mathbf{O}$ represents an all-zero matrix of dimension $4 \times 2$. It is straightforward to see that $\min \{\operatorname{rank}(\overline{\mathbf{X}}) \mid \overline{\mathbf{X}} \in \Delta \overline{\mathcal{X}}\}=4$ and hence this scheme achieves a transmit diversity order 4 .

Example 3: Let $N_{t}=8, T=1$, and $N_{S M}$ represent the number of antennas used for SM out of $N_{t}$ available antennas. Let the corresponding collection of codebooks be $\overline{\mathcal{X}}=\left\{\mathcal{X}_{i}\right\}_{i=1}^{n}$, where $n=\left(\begin{array}{c}N_{t} \\ N_{S M}\end{array}\right)$ represents the number of possible antenna subset combinations. Based on the results in [28], we have $\min \{\operatorname{rank}(\overline{\mathbf{X}}) \mid \overline{\mathbf{X}} \in \Delta \overline{\mathcal{X}}\}=N_{t}-N_{S M}+1$. Thus, the antenna subset selection scheme [25], [28] attains a transmit diversity order of $N_{t}-N_{S M}+1$.

Remark 1: Note that the residual SI is generally modeled by a Gaussian random variable [36], [40]. This is a reasonable assumption, since the channel estimation errors are often Gaussian distributed, especially when employing MMSE estimation. Thus, the effective noise at the receiver would still be Gaussian albeit at a higher power, which is equal to the sum of the powers of the AWGN and the residual SI. Thus, the diversity conditions on the transmit and receive codebooks presented in Proposition 1 and Proposition 2 hold for the case of full-duplex systems as well.

In the next section of the paper, we propose joint transmit and receive codebook (JTRCB) selection schemes and study its potential benefits in the emerging FD SM communication systems [38]-[40].

\section{Joint Transmit And Receive Codebook SELECTION AIDED FD SM}

The subject of FD communication [35]-[37] has gained significant attention in the recent past owing to its promise of nearly doubling the throughput of its simplex counterpart. In order to achieve simultaneous interference-free bidirectional communication, the FD systems employ SI cancellation in both the analog as well as the digital domains. The analog cancellation (ACL) techniques include interference cancellation based on optimum antenna placement [35], on Balun based cancellation using variable attenuators and delay elements [35], while the digital cancellation (DCL) essentially estimates the SI-leakage response and then cancels it by an FIR filter based echo-canceller [35]-[37]. The ACL plays a crucial role, since it predetermines the overall noise figure of the receive chain. Additionally, it was observed by Duarte et. al. [36] that DCL may result in error-propagation and hence should be applied selectively.

The ACL circuitry in case of a SISO FD systems is quite simple, since it involves only a set of attenuators and delay elements. However, invoking the same principle for a MIMO system would result in a quadratic complexity increase. For example, a $(2 \times 2)$ MIMO system requires four ACL blocks, as shown in Fig. 1. Similarly, an $\left(N_{t} \times N_{t}\right)$ FD MIMO system would require $N_{t}^{2}$ ACL blocks. This would impose a significant cost and power consumption. In order to overcome this impediment, a cascaded cancellation design was proposed by Bharadia and Katti [37], who exploited the cascaded 


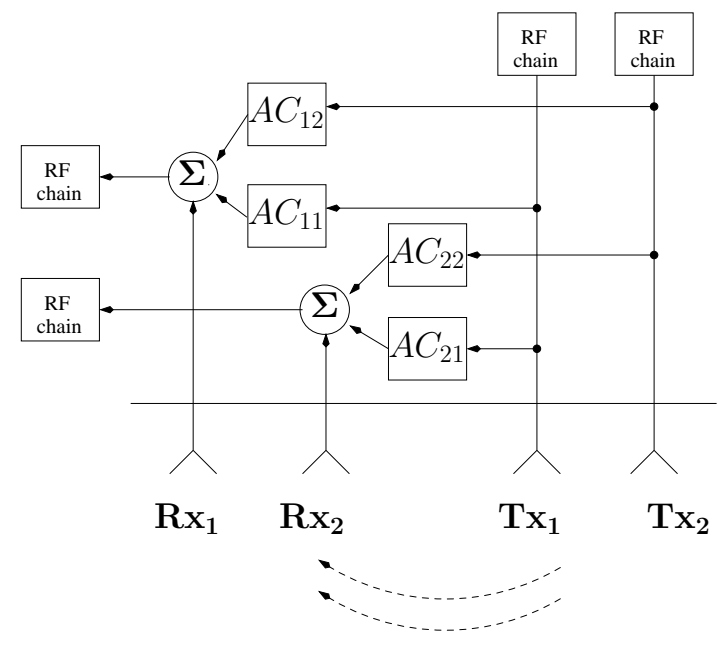

Self Interference

Fig. 1. Pictorial depiction of a $2 \times 2$ FD MIMO system, where the block $A C_{i j}$ represents the analog cancellation circuitry between the $j^{\text {th }}$ transmit and $i^{\text {th }}$ receive chain.

nature of the SI transfer function. However, this would still require a complex ACL circuitry. For more details on the cascaded cancellation design, please refer to Section 3.1 of [37]. Furthermore, it was also observed in [37] that the residual error of SI cancellation as well as the time required to tune the cancellation circuitry increase with $N_{t}$.

Figure 2 gives a pictorial portrayal of the FD SM system having two TAs and two receive antennas. Although the SM system requires only a single RF chain at the transmitter, a FD SM system would still need $N_{t}^{2}$ ACL blocks $\left(N_{r}=N_{t}\right)$, since it is not practical to have a single ACL block and reprogram it for every symbol period in order to cancel the SI. Indeed, using multiple receive antennas are imperative in case of spatial multiplexing systems, where multiple symbols are transmitted simultaneously, but they are not essential in case of SM systems. Explicitly, in case of SM systems, the multiple receive antennas are mainly used for attaining receive diversity, which can be attained with the aid of our results from Section II, despite employing a single RF chain at the receiver.

Figure 3 depicts a simple antenna subset selection aided FD SM system, where two out of three TAs are used for SM transmission and one out of three antennas is selected for reception. The antenna subset selection aided FD SM system offers the following benefits:

1) We need only $N_{S M}\left(\leq N_{t}\right)$ ACL blocks where $N_{S M}$ is the number of TAs used for SM transmission. Note that this is an order lower than $N_{t}^{2}$, which is the number of ACL blocks required by the FD MIMO system.

2) Despite its low hardware complexity, the proposed scheme attains both transmit as well as receive diversity gains, thanks to our results in Section II. In the next part of the paper, we propose several RCBs that yield full receive diversity gain, while requiring a minimum number of ACL blocks.

3) Since the residual SI cancellation error as well as the time required to tune the cancellation circuitry scale with

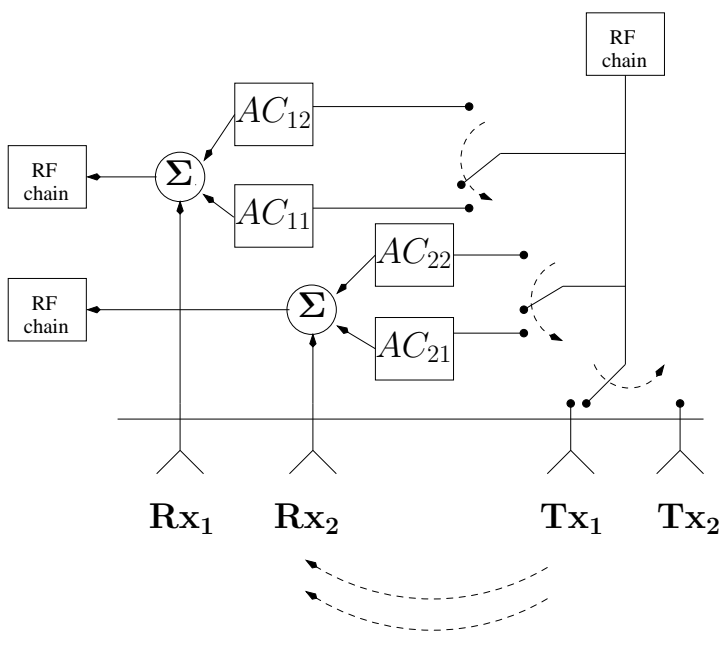

Self Interference

Fig. 2. Pictorial depiction of a $2 \times 2$ FD SM system.

TABLE I

COMPARISON OF VARIOUS SYSTEM PARAMETERS OF THE EXISTING AS WELL AS OF THE PROPOSED FD SCHEMES.

\begin{tabular}{|l|c|c|c|}
\hline & FD MIMO & FD SM & $\begin{array}{c}\text { Proposed } \\
\text { FD schemes }\end{array}$ \\
\hline \hline $\begin{array}{l}\text { No. of SI } \\
\text { cancellation blocks }\end{array}$ & $N_{r} N_{t}$ & $N_{r} N_{t}$ & $N_{S M}$ \\
\hline $\begin{array}{l}\text { Attainable } \\
\text { diversity order }\end{array}$ & $N_{r} N_{t}$ & $N_{r} N_{t}$ & $N_{r} N_{t}$ \\
\hline $\begin{array}{l}\text { No. of RF } \\
\text { switches }\end{array}$ & $*$ & $N_{r}+1$ & 2 \\
\hline $\begin{array}{l}\text { Tuning overhead } \\
\text { scaling factor }\end{array}$ & $N_{t}$ & $N_{t}$ & $N_{S M} \leq N_{t}$ \\
\hline \hline
\end{tabular}

$N_{t}$ [37], they are significantly reduced in the proposed scheme, since there is only a single active TA during each channel use and only $N_{S M} \leq N_{t}$ antennas are used for SM transmission.

Table I compares the system parameters of the proposed scheme to those of the existing FD MIMO and FD SM schemes. It is clear from Table I that the proposed schemes require only $N_{S M}$ ACL blocks in comparison to $N_{r} N_{t}$ required by the existing schemes. As a result, the power consumption in the proposed scheme is significantly reduced. Furthermore, the number of RF switches in case of the proposed scheme is only two, which enables us to ensure better synchronization between them. Furthermore, the proposed scheme has the advantage of a low tuning overhead [37] compared to the FD MIMO and FD SM schemes. Lastly, we would like to emphasize that the half-duplex SM system (HD-SM) can be easily extended to the FD SM system by employing the ACL blocks conceived for FD MIMO [37] and further improvements can be attained by employing the proposed schemes.

Again, in the next part of the paper, we propose several RCBs that allow us to exploit the full receive diversity gain without compromising the above benefits. 


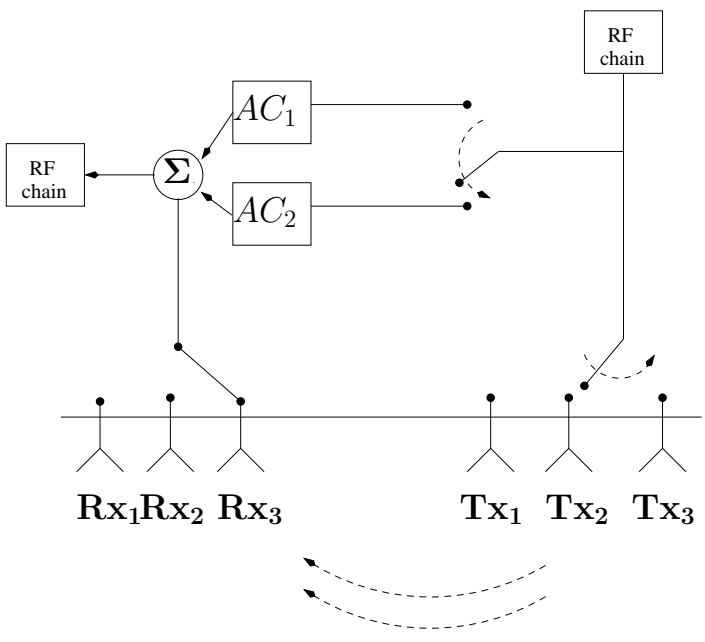

Self Interference

Fig. 3. Pictorial depiction of a $2 \times 1$ FD SM system employing receive as well as transmit antenna subset selection. Specifically, the transmit antennas indexed by 2 and 3 are used for SM transmission while the receive antenna indexed by 3 is used for reception.

\section{A. RCBs Proposed for JTRCB Aided FD SM Systems}

In this section, we first provide a simple method for obtaining $\mathbf{Z}_{j}$ 's from a given full-rank matrix $\mathbf{Q}$, while satisfying the diversity condition in Proposition 2. Later we discuss constructions based on a specific $\mathbf{Q}$ along with their advantages. Note that in this section a generalized system model is considered where $M_{r}$ may be less than $N_{r}$ by virtue of Corollary 1 .

Let $M_{r} \leq N_{r}$ represent the number of RF chains at the receiver and each $\mathbf{Z}_{j}$ represent an $\left(M_{r} \times N_{r}\right)$-element analogto-baseband processing matrix and let $\mathbf{Q} \in \mathbb{C}^{N_{r} \times N_{r}}$ represent a full-rank matrix. The proposed construction is given by

$\mathbf{Z}_{j}=\mathbf{I}_{N_{r}}\left[\left(M_{r}(j-1)+1: M_{r}(j-1)+M_{r}\right),:\right] \mathbf{Q} \in \mathbb{C}^{M_{r} \times N_{r}}$

Since $\operatorname{rank}\left(\mathbf{Z}_{j}\right)=M_{r}$ for $1 \leq j \leq N_{r} / M_{r}$, from Corollary 1 $m=N_{r} / M_{r}$ number of entries in $\mathcal{Z}$ would suffice to meet the rank condition $\operatorname{rank}(\overline{\mathbf{Z}})=N_{r}$ and hence achieve full receive diversity. Below, we discuss a few specific constructions based on $\mathbf{Q}$.

1) Receive Antenna Selection (RAS): For ease of exposition, we consider a receiver having either a single or twin RF chains. Note that the proposed RCBs can be used in receivers having multiple RF chains as well. Consider $\mathbf{Q}=\mathbf{I}_{N_{r}}$ and $M_{r}=1$. In this case, we have $\mathbf{Z}_{j}=\mathbf{I}_{N_{r}}[j,:]$ for $1 \leq j \leq N_{r}$. This scheme is analogous to the one depicted in Fig. 3. For example, consider a system having $N_{r}=4$. In this case, we have

$$
\overline{\mathbf{Z}}=\left[\begin{array}{cccc}
1 & 0 & 0 & 0 \\
\hline 0 & 1 & 0 & 0 \\
\hline 0 & 0 & 1 & 0 \\
\hline 0 & 0 & 0 & 1
\end{array}\right] \begin{gathered}
\leftarrow \mathbf{Z}_{1} \\
\leftarrow \mathbf{Z}_{2} \\
\leftarrow \mathbf{Z}_{3} \\
\leftarrow \mathbf{Z}_{4}
\end{gathered}
$$

In case of a system having $M_{r}=2 \mathrm{RF}$ chains, we still use $\mathbf{Q}=\mathbf{I}_{N_{r}}$ but $\mathbf{Z}_{j}=\mathbf{I}_{N_{r}}[(2(j-1)+1: 2(j-1)+2),:]$ for $1 \leq j \leq N_{r} / 2$. In case of the aforementioned system having
$N_{r}=4$, we have

$$
\left.\overline{\mathbf{Z}}=\left[\begin{array}{cccc}
1 & 0 & 0 & 0 \\
0 & 1 & 0 & 0 \\
\hline 0 & 0 & 1 & 0 \\
0 & 0 & 0 & 1
\end{array}\right]\right\} \mathbf{Z}_{1}
$$

Note that when $M_{r}=N_{r}$, all the antennas are used for reception, which corresponds to the conventional receive processing, in which case we have $\overline{\mathbf{Z}}=\mathbf{I}_{N_{r}}=\mathbf{Z}_{1}$.

2) Receive Combiner Selection (RCS): Consider

$$
\mathbf{Q}=\left[\mathbf{a}, \mathbf{M a}, \mathbf{M}^{2} \mathbf{a}, \ldots, \mathbf{M}^{N_{r}-1} \mathbf{a}\right],
$$

where $\mathbf{a}^{T}=\left[a_{0}, a_{1}, \ldots, a_{N_{r}-1}\right] \in \mathbb{C}^{1 \times N_{r}}$ and

$$
\mathbf{M}=\left[\begin{array}{ccccc}
0 & 0 & \cdots & 0 & 1 \\
1 & 0 & \cdots & 0 & 0 \\
0 & 1 & \cdots & 0 & 0 \\
\vdots & \vdots & \ddots & \vdots & \vdots \\
0 & 0 & \cdots & 1 & 0
\end{array}\right] \in \mathbb{R}^{N_{r} \times N_{r}}
$$

Note that $\mathbf{Q}$ in (17) is circulant [47] and the associated polynomial is given by

$$
f(x)=a_{0}+a_{1} x+\cdots+a_{N_{r}-1} x^{N_{r}-1} .
$$

Proposition 3 (Prop. 1.1 [48]): The $\operatorname{rank}(\mathbf{Q})=N_{r}-d$, where $d$ is the degree of the greatest common divisor of $x^{N_{r}}-1$ and $f(x)$.

From Proposition 3, we see that full receive diversity can only be attained if the polynomials $f(x)$ and $x^{N_{r}}-1$ do not have common divisors. Thus, the choice of the polynomial $f(x)$ is crucial for attaining the desired receive diversity gain. A simple approach to guarantee a high diversity gain is to consider polynomials $f(x)$ of lower degree. Furthermore, an additional benefit of considering lower-degree polynomials is the reduced implementation cost, as discussed later in this section.

Corollary 2: The feedback assisted MIMO system employing (4) with $\mathrm{f}(\cdot)$ given in (6) and employing RCB matrices in (14) and $\mathbf{Q}$ in (17) with $f(x)=1+x$ attains a receive diversity gain of at least $\left(N_{r}-1\right)$.

Proof: The proof is straightforward and is provided for the sake of completeness. Since the degree of the polynomial that divides $f(x)=1+x$ and $x^{N_{r}}-1$ can only be at most one, it follows from Proposition 3 that $\operatorname{rank}(\mathbf{Q})=\operatorname{rank}(\overline{\mathbf{Z}}) \geq$ $N_{r}-1$. This concludes the proof.

Note that the RCS scheme degenerates to the RAS scheme, when the polynomial $f(x)$ is a constant. Since the degree of this polynomial is zero, the RAS attains the full receive diversity gain of $N_{r}$.

Considering a system having $N_{r}=4, M_{r}=1$ and $\mathbf{Q}$ in (17) associated with $f(x)=1+x$, we have

$$
\overline{\mathbf{Z}}=\left[\begin{array}{cccc}
1 & 0 & 0 & 1 \\
\hline 1 & 1 & 0 & 0 \\
\hline 0 & 1 & 1 & 0 \\
\hline 0 & 0 & 1 & 1
\end{array}\right] \begin{gathered}
\leftarrow \mathbf{Z}_{1} \\
\leftarrow \mathbf{Z}_{2} \\
\leftarrow \mathbf{Z}_{3} \\
\leftarrow \mathbf{Z}_{4}
\end{gathered}
$$

It is clear from (20) that each of the RCB entries $\mathbf{Z}_{j}$ combines signals received from two antenna ports. For instance, $\mathbf{Z}_{1}$ 
combines the signals received from antenna one and antenna four, whereas $\mathbf{Z}_{2}$ combines that of one and two, and so on and so forth. This RCB achieves a diversity gain of three, since we have $\operatorname{rank}(\mathbf{Q})=3$. When $N_{r}$ is small, there can be a noticeable performance loss with respect to the scheme achieving full receive diversity gain. The following proposition gives a simple method of selecting polynomials that achieve full diversity.

Corollary 3: The feedback-assisted MIMO system employing (4) with $f(\cdot)$ given in (6) and employing the RCB matrices in (14) and $\mathbf{Q}$ in (17) attains a receive diversity gain of $N_{r}$, if we have $f(x)=\boldsymbol{\Phi}_{k}(x)$, provided that $1<k<N_{r}$ does not divide $N_{r}$.

Proof: The proof is straightforward and is provided for the sake of completeness. For any given $N_{r}$, we have

$$
\begin{aligned}
x^{N_{r}}-1 & =\prod_{1 \leq l \leq N_{r}, l \mid N_{r}} \boldsymbol{\Phi}_{l}(x), \\
& =\boldsymbol{\Phi}_{1}(x) \boldsymbol{\Phi}_{l_{1}}(x) \boldsymbol{\Phi}_{l_{2}}(x) \cdots \boldsymbol{\Phi}_{N_{r}}(x),
\end{aligned}
$$

where each of the elements in $\left\{l_{1}, l_{2}, \ldots\right\}$ divides $N_{r}$. Let $f(x)=\boldsymbol{\Phi}_{k}(x)$ such that $1<k<N_{r}$ and $N_{r}$ is not a multiple of $k$. Since $\boldsymbol{\Phi}_{m}(x)$ and $\boldsymbol{\Phi}_{n}(x)$ are co-prime for $m \neq n$, it follows that $x^{N_{r}}-1$ and $f(x)$ are co-prime. This concludes the proof.

The following example illustrates the significance of Corollary 3.

Example 4: Consider a system having $N_{r}=6$ and $M_{r}=1$. If we consider the polynomial $f(x)=1+x$, we have

$$
\overline{\mathbf{Z}}=\left[\begin{array}{cccccc}
1 & 0 & 0 & 0 & 0 & 1 \\
\hline 1 & 1 & 0 & 0 & 0 & 0 \\
\hline 0 & 1 & 1 & 0 & 0 & 0 \\
\hline 0 & 0 & 1 & 1 & 0 & 0 \\
\hline 0 & 0 & 0 & 1 & 1 & 0 \\
\hline 0 & 0 & 0 & 0 & 1 & 1
\end{array}\right] \begin{gathered}
\leftarrow \mathbf{Z}_{1} \\
\leftarrow \mathbf{Z}_{2} \\
\leftarrow \mathbf{Z}_{3} \\
\leftarrow \mathbf{Z}_{4} \\
\leftarrow \mathbf{Z}_{5} \\
\leftarrow \mathbf{Z}_{6}
\end{gathered}
$$

where $\operatorname{rank}(\overline{\mathbf{Z}})=N_{r}-1=5$. Note that $x^{6}-1=$ $\boldsymbol{\Phi}_{1}(x) \boldsymbol{\Phi}_{2}(x) \boldsymbol{\Phi}_{3}(x) \boldsymbol{\Phi}_{6}(x)$ and the choice of $f(x)=\boldsymbol{\Phi}_{4}(x)=$ $1+x^{2}$ yields

$$
\overline{\mathbf{Z}}=\left[\begin{array}{cccccc}
1 & 0 & 0 & 0 & 1 & 0 \\
\hline 0 & 1 & 0 & 0 & 0 & 1 \\
\hline 1 & 0 & 1 & 0 & 0 & 0 \\
\hline 0 & 1 & 0 & 1 & 0 & 0 \\
\hline 0 & 0 & 1 & 0 & 1 & 0 \\
\hline 0 & 0 & 0 & 1 & 0 & 1
\end{array}\right] \begin{gathered}
\leftarrow \mathbf{Z}_{1} \\
\leftarrow \mathbf{Z}_{2} \\
\leftarrow \mathbf{Z}_{3} \\
\leftarrow \mathbf{Z}_{4} \\
\leftarrow \mathbf{Z}_{5} \\
\leftarrow \mathbf{Z}_{6}
\end{gathered}
$$

where $\operatorname{rank}(\overline{\mathbf{Z}})=N_{r}=6$. Note that although both the codebooks in (23) and (24) combine signals from two antenna ports, the specific choice of the antenna ports determines the attainable diversity gain.

It is important to note that the coefficients in the Cyclotomic polynomials are from the set $\{1,-1,0\}$ up to $\boldsymbol{\Phi}_{105}(x)$. Thus, using simple combiners and signal inverters is sufficient to implement the receive codebook processing for up to $N_{r} \leq$ 104. Table II in the next page provides some examples of Cyclotomic polynomials along with the achievable receive diversity order. A general method for finding the coefficients of any Cyclotomic polynomial can be found in [49].
TABLE II

EXAMPLES OF CYCLOTOMIC POLYNOMIALS AND THEIR ACHIEVABLE DIVERSITY ORDER.

\begin{tabular}{|c|c|c|c|c|}
\hline$N_{r}$ & $N_{c}$ & $M_{r}$ & $\boldsymbol{\Phi}(x)$ & $\begin{array}{c}\text { Receive } \\
\text { diversity } \\
\text { order }\end{array}$ \\
\hline \hline 4 & 2 & 1 & $\boldsymbol{\Phi}_{2}(x)=1+x$ & 3 \\
\hline & 3 & 1 & $\boldsymbol{\Phi}_{3}(x)=1+x+x^{2}$ & 4 \\
\hline \hline 8 & 2 & 1 & $\boldsymbol{\Phi}_{2}(x)=1+x$ & 7 \\
\hline & 3 & 1 & $\boldsymbol{\Phi}_{3}(x)=1+x+x^{2}$ & 8 \\
\hline & 5 & 1 & $\boldsymbol{\Phi}_{5}(x)=1+x+x^{2}+x^{3}+x^{4}$ & 8 \\
\hline & 6 & 1 & $\boldsymbol{\Phi}_{6}(x)=1-x+x^{2}$ & 8 \\
\hline \hline 16 & 2 & 1 & $\mathbf{\Phi}_{2}(x)=1+x$ & 15 \\
\hline & 7 & 1 & $\boldsymbol{\Phi}_{7}(x)=\sum_{i=0}^{6} x^{i}$ & 16 \\
\hline & 12 & 1 & $\boldsymbol{\Phi}_{12}(x)=1-x^{2}+x^{4}$ & 16 \\
\hline & 13 & 1 & $\boldsymbol{\Phi}_{13}(x)=\sum_{i=0}^{12} x^{i}$ & 16 \\
\hline \hline
\end{tabular}

3) Receive Combiner Selection with Phase Shifters (RCS$P S)$ : Consider $\mathbf{Q}$ such that $|\mathbf{Q}(i, j)|=1$ for $1 \leq i, j \leq N_{r}$. Note that the resultant $\mathbf{Z}_{j}$ 's based on (14) require analog phase shifters for their implementation. A compelling choice of $\mathbf{Q}$ would be the DFT matrix, which satisfies the full rank condition. That is, with $\mathbf{Q}=\mathbf{F}_{N_{r}}$ we have $\operatorname{rank}(\mathbf{Q})=$ $\operatorname{rank}(\overline{\mathbf{Z}})=N_{r}$. Considering $N_{r}=4$ and $M_{r}=1$, we have

$$
\overline{\mathbf{Z}}=\left[\begin{array}{cccc}
1 & 1 & 1 & 1 \\
\hline 1 & -j & -1 & j \\
\hline 1 & -1 & 1 & -1 \\
\hline 1 & j & -1 & -j
\end{array}\right] \begin{gathered}
\leftarrow \mathbf{Z}_{1} \\
\leftarrow \mathbf{Z}_{2} \\
\leftarrow \mathbf{Z}_{3} \\
\leftarrow \mathbf{Z}_{4}
\end{gathered}
$$

where $j=\sqrt{-1}$. As a benefit of the appealingly simple implementation and high energy efficiency of the RAS and RCS schemes, we restrict our study to these two schemes in the rest of the paper.

\section{B. Impact of SI on Receiver Processing}

In this section, we briefly discuss our model of the residual error after ACL due to SI in the case of RAS and RCS aided FD SM systems. Since the SM system employs only a single TA in each channel use, the SI observed at each receive antenna is only due to a single transmitted symbol. Furthermore, in case of the RAS scheme only a few antennas are used for reception, as seen in Fig. 3. In our simulation studies, we consider RAS scheme using $M_{r}=1$ and $M_{r}=2$. In both of these cases the residual error after analog cancellation is modeled as $\sqrt{\rho_{S I}} s h$, where $s$ is the transmitted symbol, $h \sim \mathcal{C N}(0,1)$ is the channel coefficient between the active TA and the selected receive antenna(s) of the FD SM system and $\rho_{S I}$ is the residual SI-to-noise ratio (SINR). Note that our residual SI model is in accordance with the assumptions found in the existing literature [39], [40]. In case of the RCS scheme, where the signals received by multiple antennas are combined, the residual error after analog cancellation is modeled as $\sqrt{N_{c} \rho_{S I}} s h$, where $h \sim \mathcal{C N}(0,1)$ is the effective channel coefficient between the active TA and the combiner output, while $N_{c}$ is the number of antenna ports used for signal combining. Note that the effective SINR in this case is modeled to be $N_{c}$ times higher compared to the RAS scheme, 


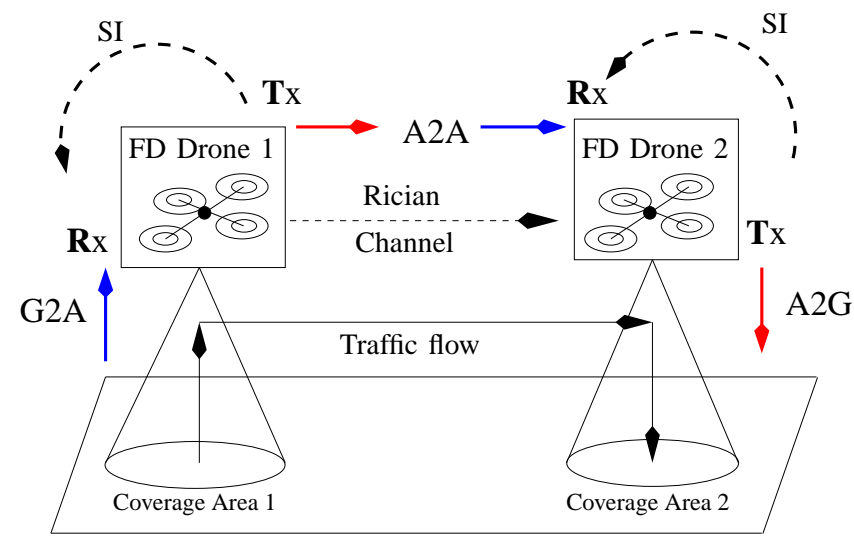

Fig. 4. Pictorial representation of a triple-hop relay network where both the drones act as relays and operate in the FD mode. The three information hops correspond to G2A link, A2A link followed by A2G link. The A2A link is modeled as the Rician channel with parameter $K$, where as the G2A and A2G links are modeled as Rayleigh channels.

since only a single ACL block is used for canceling the $N_{c}$ combined interference components. We find it important to mention that the modeling of SI in the context of SM systems needs special attention, which has not been explicitly studied in the literature. Furthermore, note that the results in Proposition 1 and Proposition 2 hold even in the case of FD systems. However, in case of FD systems, instead of $\rho$ being the effective SNR, $\rho /\left(1+\rho_{S I}\right)$ or $\frac{\rho}{1+N_{c} \rho_{S I}}$ will be the effective SNR. Thus, the proposed RCBs in the FD system attain the same diversity gain as attained in their simplex counterparts, but at a relatively higher SNR.

\section{Application of JTRCB aided FD SM Systems in Drone Communication}

Recently, there has been a significant interest in drone aided communication systems [41]-[45]. Owing to their easy deployability and wide range of applications, drones are becoming increasingly popular, especially in the areas of search and rescue operations, providing temporary network coverage in large gatherings such as sports events [44], providing emergency network coverage in the disaster-struck areas [45] etc. In this paper, we consider FD SM for establishing communication between drones, i.e. air-to-air (A2A) links, as well as between the drones and the terrestrial terminals, i.e. ground-to-air (G2A) and air-to-ground (A2G) links. Specifically, we propose a multi-hop relay system, where each drone acts as a relay node that employs a RAS/RCS aided FD SM scheme.

Figure 4 gives a pictorial portrayal of a triple-hop network, where two full-duplex drones (FD Drone 1 and FD Drone 2) act as relays for establishing a communication link between two geographically distant coverage areas (Coverage Area 1 to Coverage Area 2 ). Each of the FD drones transmit as well as receive data simultaneously and facilitate information flow from Coverage Area 1 to Coverage Area 2, as shown in Fig. 4. In the next part of the paper, we provide our simulation results, where we consider the network topology presented in Fig. 4.

There are several advantages in invoking FD SM systems for drone communications:
1) The FD communication offers nearly doubled throughput compared to its simplex counterpart, which enables us to address the commonly seen congestion issue in drone deployments, where a large number of users simultaneously access the network.

2) Since small drones are battery operated, they have to be energy-efficient. Thus, the SM becomes an ideal MIMO transmission scheme owing to its reduced hardware complexity and superior energy efficiency [16] compared to other MIMO schemes, such as BLAST.

3) Furthermore, the RAS/RCS aided FD SM system further reduces the hardware and power requirements of the system, hence further improving the energy efficiency of the system. In addition to the improved energy efficiency, the RCS aided SM systems avoid the ill effects of strong line-of-sight (LoS) channels, which are common in A2A links.

\section{Simulation Results and Discussions}

Simulation scenario: In all our simulations, we have employed at least $10^{t+1}$ bits for evaluating a bit error rate (BER) of $10^{t}$. We use the following notations for denoting the RAS as well as the RCS schemes proposed in this paper. The RAS scheme associated with various system parameters is denoted by $\operatorname{RAS}\left(N_{r}, M_{r}, L\right)$, where $L$ denotes the size of the QAM signal set, while the RCS scheme is denoted by $R C S\left(N_{r}, N_{c}, L\right)$, where $N_{c}$ denotes the number of combiners. The transmission parameters of the SM system are $N_{t}=6$ and $N_{S M}=4$, unless stated otherwise. In all our simulations, we consider $N_{c}=2$, which corresponds to the polynomial of $f(x)=1+x$, unless stated otherwise. The receiver is assumed to have perfect CSIR and employs ML decoding.

\section{A. Validation of Receive Diversity Gain of RAS and RCS Codebooks}

In order to validate the theoretical claims on the achievable diversity gain of our RAS and RCS codebooks, let us consider a point-to-point SM transmission scheme having no transmit diversity gain. Let the transmission parameters be $N_{t}=N_{S M}=4$ and use a 4-QAM signal set, which ensure that any diversity gain attained is only due to the receive processing. Figure 5 provides the BER performance of various RAS and RCS schemes along with the reference curves of the form $a / S N R^{r}$ for comparison, where $a$ is a positive constant. Specifically, Fig. 5(a) compares the attainable diversity order of the $R A S(2,1,4), R A S(4,1,4)$ and $R A S(4,2,4)$ schemes. It can be readily seen from Fig. 5(a) that the proposed RAS codebooks indeed succeed in attaining the full receive diversity gain of $N_{r}$. Figure 5(b) compares the attainable diversity order of $\operatorname{RCS}(2,2,4)$, and $\operatorname{RCS}(4,3,4)$, which employ $f(x)=$ $1+x$ and $f(x)=1+x+x^{2}$ respectively. It can be seen from Fig. 5(b) that the proposed RCS codebooks attain the expected diversity gain as per our theoretical claims in Corollary 2 and Corollary 3. 

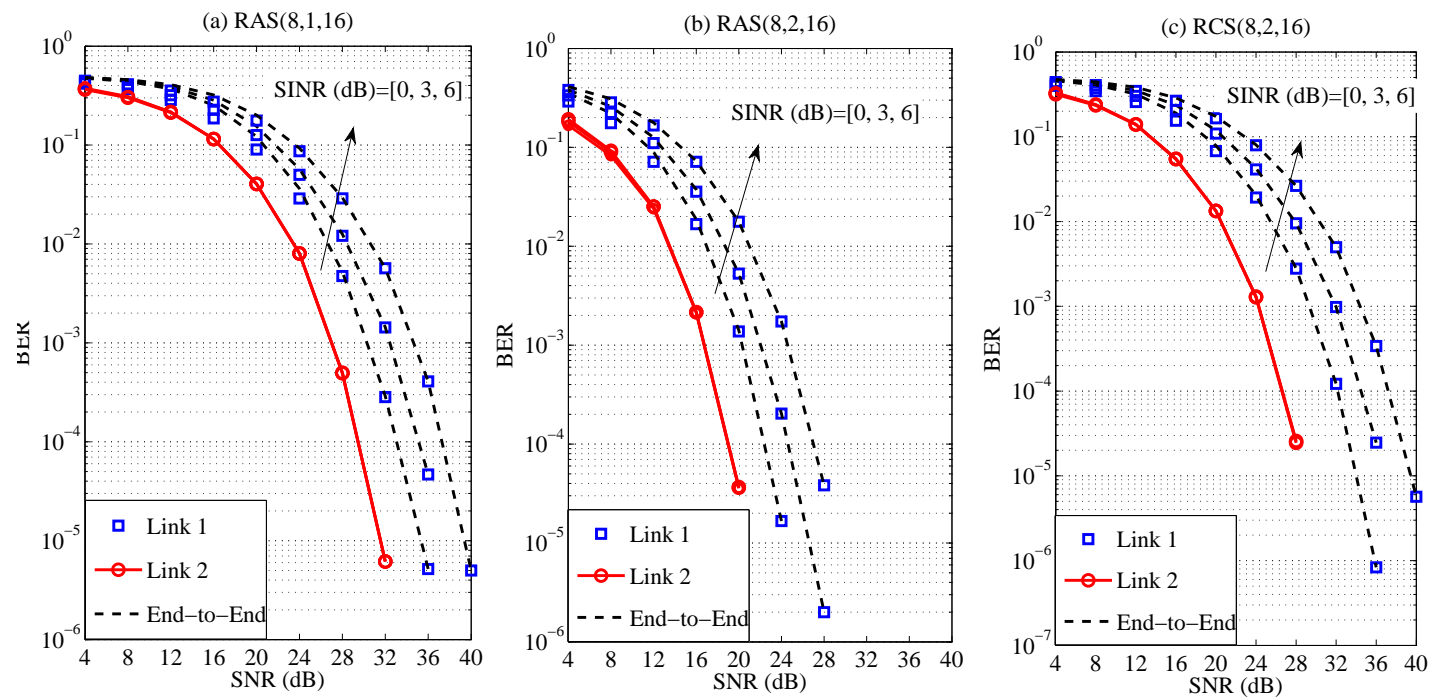

Fig. 6. BER performance curves of the dual-hop relay system employing $R A S(8,1,16), R A S(8,2,16)$ and $R C S(8,2,16)$ over both communication links. The performance curves correspond to three SINR values of $0 \mathrm{~dB}, 3 \mathrm{~dB}$, and $6 \mathrm{~dB}$. The diversity order attained by the RAS schemes is twenty four, whereas that attained by the RCS scheme is twenty one. All the schemes are assumed to operate at a spectral efficiency of 6 bpcu.
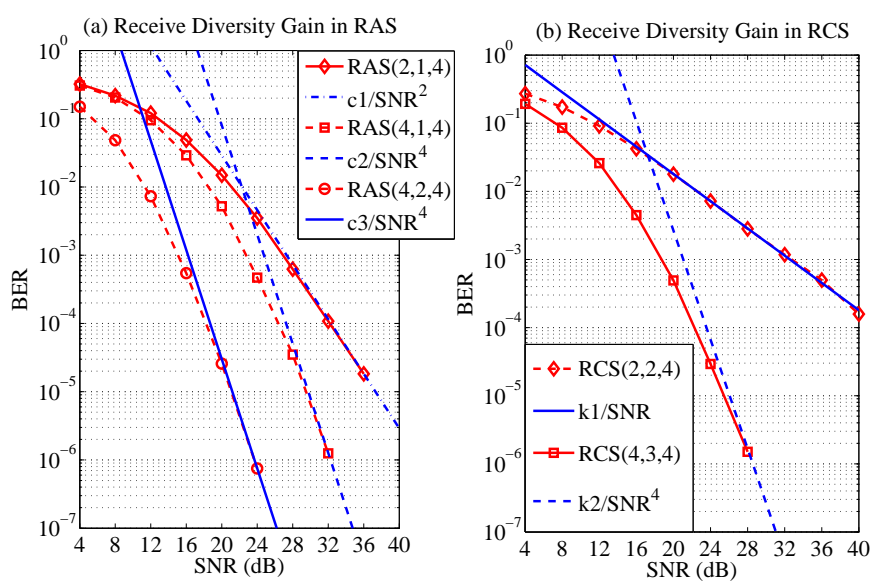

Fig. 5. The achievable diversity gain of the RAS and RCS codebooks in an SM scheme having $N_{t}=N_{S M}=4$ and employing 4-QAM signal set. The reference curves of the form $a / S N R^{r}$ are provided for easily verifying the diversity order attained by the proposed codebooks. The diversity order attained by the RAS schemes in Plot(a) are two and four, whereas that attained by the RCS schemes in Plot(b) are one and four. All the schemes are assumed to operate at a spectral efficiency of $4 \mathrm{bpcu}$.

\section{B. Performance of RAS and RCS Codebooks in Dual-Hop Relay Network}

Let us consider a dual-hop relay network, where the source node is equipped with $N_{t}=6, N_{S M}=4$ and the destination node is equipped with $N_{r}=8$. The relay node is assumed to be employing FD SM having $N_{r}=8, N_{t}=6$ and $N_{S M}=4$. Note that the antennas used for transmission and the reception at the relay node do not have to belong to distinct sets. That is, when using RAS or RCS codebooks, only one or two antennas are utilized out of the full set of $N_{r}$ antennas. Thus, the remaining antennas can be used for SM transmission. In the above scenario, after the choice of RCB which selects one or two antennas out of $N_{r}=8$ antennas, the remaining six antennas can be used for transmission, thus reducing the effective number of antennas required for both transmission as well as reception. Furthermore, let us denote the communication link between the source node and the relay node as Link 1 and that between the relay node and the destination node as Link 2. Figure 6 gives the achievable BER performance for three values of SINR over Link 1, Link 2 and the effective error rate denoted by End-to-End. The following observations can be made from Fig. 6:

1) the End-to-End BER performance is dictated by the performance of Link 2 in all the three schemes considered;

2) the increase in SINR results in a proportional increase in the performance degradation. This is evident from each of the plots in Fig. 6, where the Link 1 and End-to-End BER performance degrade as the SINR is increased from $0 \mathrm{~dB}$ to $6 \mathrm{~dB}$;

3) the $\operatorname{RAS}(8,2,16)$ scheme which requires two receive RF chains, attains a significantly better performance compared to the other two schemes, which rely on a single receive RF chain;

4) although $R A S(8,1,16)$ and $R C S(8,2,16)$ only use a single receive RF chain, the Link 2 BER performance of the latter scheme is better than that of the former.

Note that although the Link 2 BER performance of $R C S(8,2,16)$ is better than that of $R A S(8,1,16)$, the Endto-End BER performance is dominated by the Link 1 BER performance. Figure 7 provides the Link 1 as well as the End-toEnd BER performance of $R A S(8,1,16)$ and $\operatorname{RCS}(8,2,16)$ for various values of SINR, when the operating SNR is fixed to $32 \mathrm{~dB}$. It is evident from Fig. 7 that the $\operatorname{RCS}(8,2,16)$ gives a better BER performance than the $\operatorname{RAS}(8,1,16)$, when the SINR is low. However, as the SINR increases, it can be observed from Fig. 7 that the BER performance of 
$R C S(8,2,16)$ approaches that of $R A S(8,1,16)$.

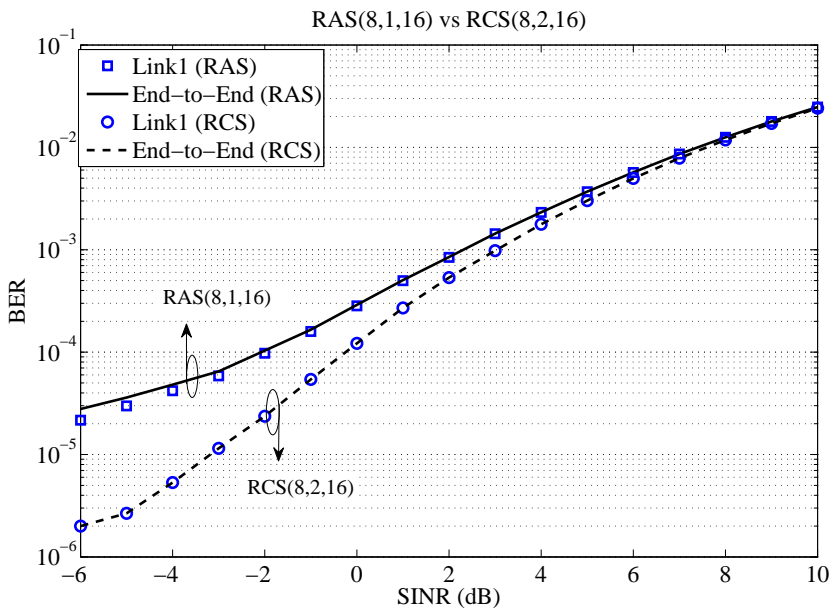

Fig. 7. Comparison of BER as a function of SINR in the dual-hop relay system employing $\operatorname{RAS}(8,1,16)$ and $\operatorname{RAS}(8,2,16)$.

Figure 8 compares the End-to-End BER performance of the dual-hop relay system employing $\operatorname{RAS}(8,1, M)$, $R A S(8,2, M)$ and $R C S(8,2, M)$ in $\mathrm{HD}$ as well as FD modes. In both the modes, all the schemes are assumed to be operating at a spectral efficiency of $3 \mathrm{bpcu}$. The FD SM system is assumed to have an SINR of 0dB. It is evident from Fig. 8 that the FD SM system outperforms its HD counterpart in all the schemes considered. Specifically, an SNR gain of about $8 \mathrm{~dB}$ is observed in case of $\operatorname{RAS}(8,1, M)$ and $\operatorname{RCS}(8,2, M)$ schemes and about $3 \mathrm{~dB}$ in case of $\operatorname{RAS}(8,2, M)$.
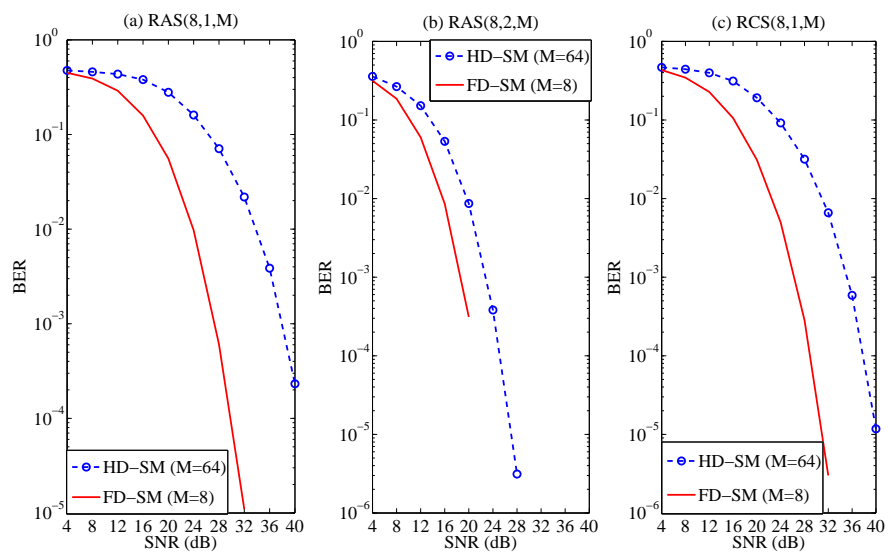

Fig. 8. End-to-End BER performance curves of the dual-hop relay system employing $\operatorname{RAS}(8,1, M), \operatorname{RAS}(8,2, M)$ and $R C S(8,2, M)$ over both communication links. The performance curves correspond to the HD-SM as well as the FD-SM systems. All the schemes are assumed to operate at a spectral efficiency of $3 \mathrm{bpcu}$. In case of FD SM system, the SINR is assumed to be $0 \mathrm{~dB}$

Figure 9 compares the End-to-End BER performance of the dual-hop relay system employing $R A S(8,1,16)$, $R A S(8,2,16)$ and $R C S(8,2,16)$, when operating in Ricean channels having $K$ factors of $0 \mathrm{~dB}$ and $20 \mathrm{~dB}$. It is evident from Fig. 9 that the BER curves of all the schemes attain the same diversity order even in the case of strong LoS channels.
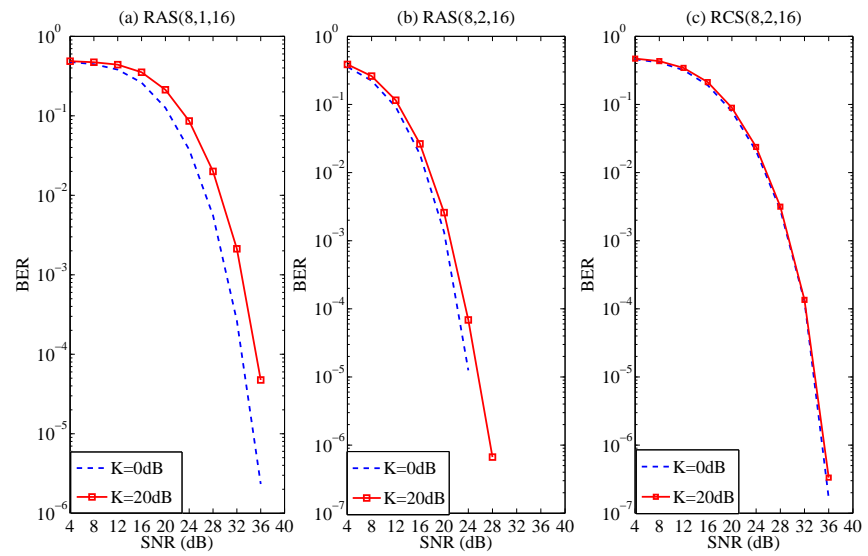

Fig. 9. End-to-End BER performance curves of the dual-hop relay system employing $R A S(8,1,16), R A S(8,2,16)$ and $\operatorname{RCS}(8,2,16)$ over both communication links. The two performance curves in each of the plots correspond to the Ricean channels having $K$ factor of $0 \mathrm{~dB}$ and $20 \mathrm{~dB}$. All the schemes are assumed to operate at a spectral efficiency of $6 \mathrm{bpcu}$ and have an SINR of OdB. (a) Link1 with RAS $(8,2,16)$ Scheme

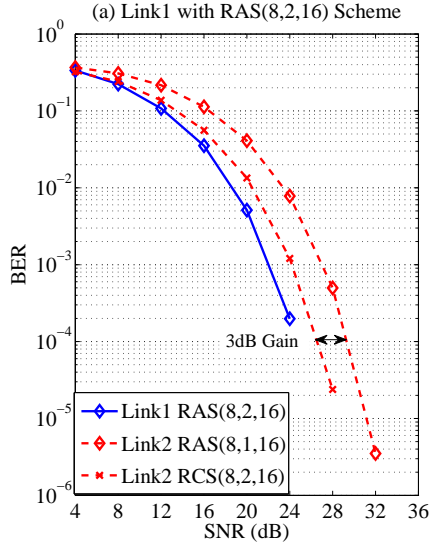

(b) Link1 with $\operatorname{RCS}(8,2,16)$ Scheme

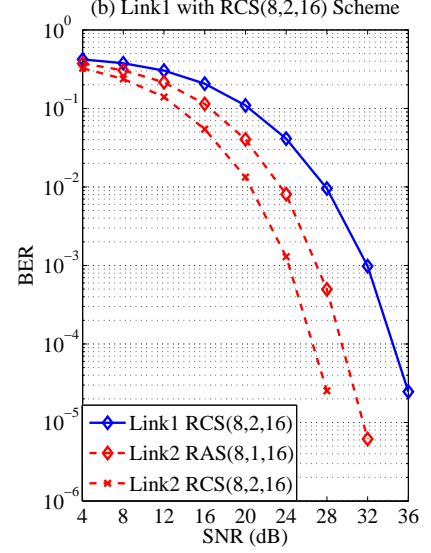

Fig. 10. Comparison of the BER performance in the two cases where $R A S(8,2,16)$ and $R C S(8,2,16)$ are employed over Link 1 in conjunction with $R A S(8,1,16)$ and $R C S(8,2,16)$ employed over Link 2 . The diversity order attained by the RAS schemes is twenty four, whereas that attained by the RCS scheme is twenty one. Both the schemes are assumed to operate at a spectral efficiency of $6 \mathrm{bpcu}$.

So far, we considered both the links to be employing the same RCB (RAS or RCS). Let us now study the performance of the dual-hop relay network, when different RCB schemes are employed over Link 1 and Link 2. Let us consider two cases, where communication over Link 1 relies either on $\operatorname{RAS}(8,2,16)$ or on $\operatorname{RCS}(8,2,16)$. For each of these choices, the BER performance of Link 2 is studied considering $R A S(8,1,16)$ and $R C S(8,2,16)$. Figure 10 provides the attainable BER performance in the above scenarios. Specifically, Fig. 10(a) provides the BER performance, when $\operatorname{RAS}(8,2,16)$ is employed over Link 1 and Fig. 10(b) corresponds to the case, where $\operatorname{RCS}(8,2,16)$ is employed. It can be readily seen from Fig. 10(a) that the BER performance of Link 2 can be improved by about $3 \mathrm{~dB}$ upon 
employing $R C S(8,2,16)$ instead of $R A S(8,1,16)$. It is worth mentioning that this improvement is attained by additionally employing a combiner, while retaining the benefit of a single $\mathrm{RF}$ chain, as in the case of $R A S(8,1,16)$. Since the Endto-End BER performance is dictated by the Link 2 BER performance, we see an overall improvement in the BER. Observe from Fig. 10(b) that the Link 1 BER dominates the overall performance and hence the improvement in the Link 2 BER performance is not helpful. Thus, it is important to ensure that high-integrity schemes are employed over the source-torelay link and the low-complexity schemes are restricted to the relay-to-destination link.

Note that the $3 \mathrm{~dB}$ gain in the overall performance observed in the case, where $\operatorname{RAS}(8,2,16)$ is employed over Link 1 holds for other combinations of Link 1 and Link 2 SNR values as well. Figure 11 (see next page) gives the 2-D BER performance plot of Link 1 and Link 2 for the cases where $R A S(8,1,16)$ and $R C S(8,2,16)$ are employed over Link 2. Comparing Fig. 11(b) to Fig. 11(a), it can be readily seen that there is a $3 \mathrm{~dB}$ gain in the Link $2 \mathrm{SNR}$ for a wide range of Link 1 SNR values. It is important to note that the $3 \mathrm{~dB}$ advantage nearly doubles the range of the relay node without requiring additional transmission power or computationally intensive receive processing.

\section{Application of RAS and RCS in Drone Communication}

Consider the scenario depicted in Fig. 4, where both the drones act as FD relays. Since both the drones have strong line-of-sight (LoS) signal, we assume the A2A channel to be Rician, while the A2G and G2A channels to be Rayleigh. In order to understand the impact of the LoS component, we first study the BER performance of various proposed RCBs as a function of the LOS signal power quantified by the $K$-factor measured in $\mathrm{dB}$ with respect to the signal power in the non-deterministic component. We consider the simple dual-hop network discussed earlier. Figure 12 compares the attainable BER performance of $R A S(8,1,16), R A S(8,2,16)$ and $\operatorname{RCS}(8,2,16)$ as a function of $K$, when operating at an SNR of $32 \mathrm{~dB}, 24 \mathrm{~dB}$ and $32 \mathrm{~dB}$, respectively. It is evident from Fig. 12 that the RAS codebooks suffer from a performance degradation as $K$ increases, while the RCS scheme remains relatively robust. This is due to the reduction in the effective LoS signal strength owing to the combiner, which combines two LoS components having random phase offsets and hence statistically eliminating the detrimental effect of a high $K$. Thus, the RCS codebooks not only give a better performance compared to the RAS codebooks as observed in Fig. 7, but are also quite robust to the ill effects of the strong LoS signals.

Owing to the strict space limitations of the journal, we restrict our study of the proposed RCBs to two system configurations. Let the first configuration (Config. 1) correspond to the case, where $\operatorname{RAS}(8,1,16)$ are employed over all the three communication links of Fig. 4. The second configuration (Config. 2) is chosen to be the same as Config. 1, except that $\operatorname{RCS}(8,2,16)$ is employed over Link 2, i.e. the A2A link between the drones. Figure 13 compares the Link 2 and Endto-End BER performance of both configurations mentioned

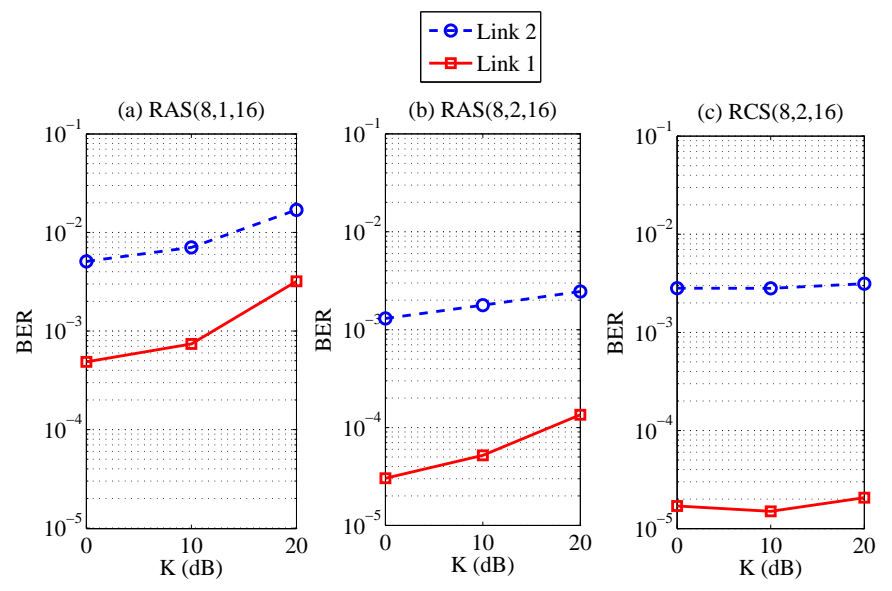

Fig. 12. Variation of BER performance of $R A S(8,1,16), R A S(8,2,16)$ and $R C S(8,2,16)$ as a function of $K$, when operating at an SNR of $32 \mathrm{~dB}$, $24 \mathrm{~dB}$ and $32 \mathrm{~dB}$, respectively. All the schemes are assumed to operate at a spectral efficiency of $6 \mathrm{bpcu}$.

above. It is evident from Fig. 13, that Config. 2 outperforms Config. 1, thanks to the robustness of the RCS codebooks to strong LoS signals. Specifically, at a BER of $10^{-4}$ Config. 2 achieves an SNR gain of about $2.5 \mathrm{~dB}$ w.r.t. Config. 1. Thus, we can infer that in a multi-hop drone system, where several drones act as relays, it is essential to consider combiner based codebooks to mitigate the detrimental effects of strong LoS signals.

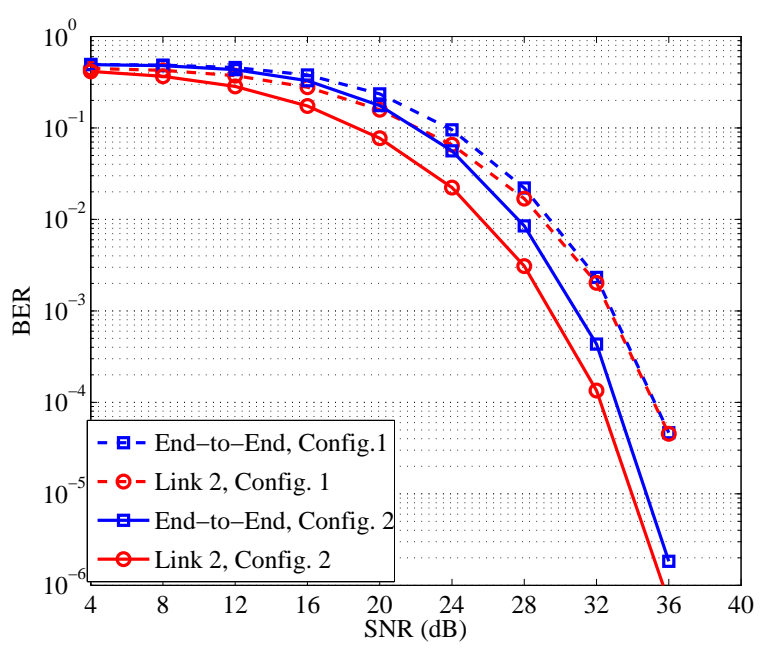

Fig. 13. Comparison of BER performance in Config. 1 to that of Config. 2 in the triple-hop drone network in Fig. 4.

\section{Conclunsions}

We have considered the family of feedback-assisted MIMO systems employing both transmit as well as receive codebooks and derived the attainable transmit and receive diversity order. Specifically, the necessary and sufficient conditions on the TCBs and RCBs to be met for attaining full diversity gain 
(a) Link 2 RAS $(8,1,16)$

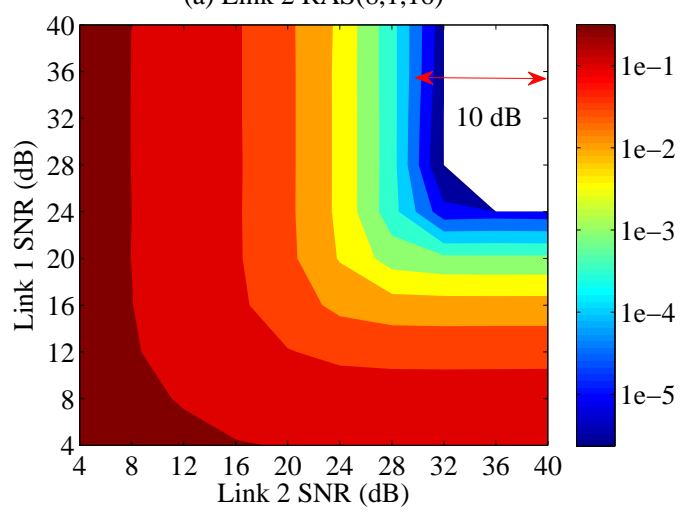

(b) Link 2 RCS $(8,2,16)$

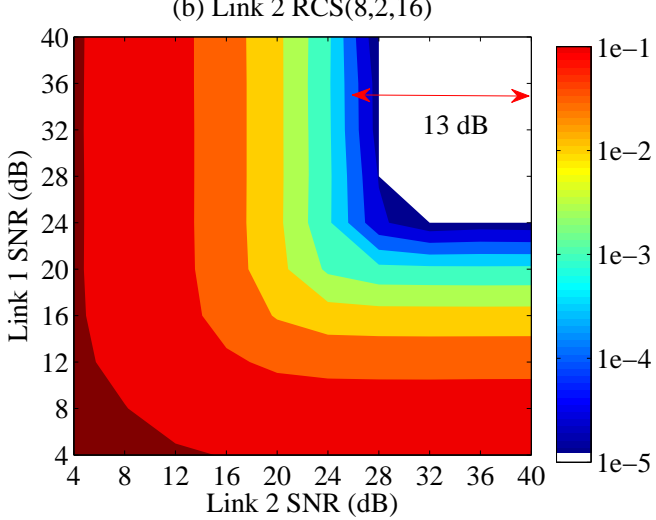

Fig. 11. Comparison of the BER performance in the two cases where $R A S(8,1,16)$ and $R C S(8,2,16)$ employed over $\operatorname{Link} 2$ while $R A S(8,2,16)$ is employed over Link 1.

were obtained. Based on the conditions derived, several fulldiversity RCBs were designed by exploiting the properties of circulant matrices embedding Cyclotomic polynomials. Furthermore, the proposed codebooks were shown to significantly reduce the hardware complexity of the interference cancellation circuitry when employed in full-duplex SM systems. The performance of the proposed codebooks was studied in drone communication applications, where several drones act as FD relays. Our simulation results have shown that the proposed codebooks exhibit robustness both to the selfinterference encountered in full-duplex systems, and to strong LoS signals. This resulted in an improved BER performance without requiring additional transmission power.

\section{APPENDIX A}

\section{ProOF OF Proposition 1}

Proof: Let the optimal TCB $\mathcal{X}_{i^{*}}$ and receive processing matrix $\mathbf{Z}_{j^{*}}$ be as in (4). When using the optimal TCB and the receive processing matrix, the pairwise error probability (PEP) between any two distinct transmit matrices in $\mathcal{X}_{i^{*}}$ indexed by $i_{1}$ and $i_{2}$ is given by

$$
\begin{aligned}
& \begin{array}{l}
\begin{aligned}
\operatorname{PEP}\left(\mathbf{X}_{i_{1}} \rightarrow \mathbf{X}_{i_{2}} \mid \mathbf{H}\right) & =Q\left(\frac{\sqrt{\rho / M_{t}}}{2} \| \mathbf{Z}_{j^{*}} \mathbf{H}\left(\mathbf{X}_{i_{1}}-\mathbf{X}_{i_{2}}\right)\right. \\
& \leq \frac{1}{2} \exp \left(-\frac{\rho}{4 M_{t}} \| \mathbf{Z}_{j^{*}} \mathbf{H}\left(\mathbf{X}_{i_{1}}-\right.\right.
\end{aligned} \\
\text { Then we have } \\
\qquad\left\|\mathbf{Z}_{j^{*}} \mathbf{H}\left(\mathbf{X}_{i_{1}}-\mathbf{X}_{i_{2}}\right)\right\|^{2} \geq \underset{m}{\| \mathbf{Z}_{j^{*}}} \mathbf{H X}_{\min }\left(j^{*}, i^{*}\right) \|_{F}^{2},
\end{array} \\
& \geq \frac{1}{m n} \sum_{j=1}^{m}\left\|\mathbf{Z}_{j} \mathbf{H} \overline{\mathbf{X}}_{j}\right\|_{F}^{2} .
\end{aligned}
$$

The inequality in (29) follows from the fact that $\left(\mathbf{Z}_{j^{*}}, \mathcal{X}_{i^{*}}\right)$ corresponds to the maximum ED among the elements in the set $\left\{\left(\mathbf{Z}_{j}, \mathcal{X}_{i}\right) \mid 1 \leq i \leq n, 1 \leq j \leq m\right\}$. Furthermore, we have

$$
\left\|\mathbf{Z}_{j} \mathbf{H} \overline{\mathbf{X}}_{j}\right\|_{F}^{2}=\operatorname{Tr}\left(\mathbf{Z}_{j} \mathbf{H} \overline{\mathbf{X}}_{j} \overline{\mathbf{X}}_{j}^{H} \mathbf{H}^{H} \mathbf{Z}_{j}^{H}\right)
$$

which can be equivalently written as

$\operatorname{vec}\left(\mathbf{H}^{H}\right)^{H}\left[\left(\mathbf{Z}_{j}^{T} \otimes \mathbf{I}_{N_{t}}\right)\left(\mathbf{I}_{N_{r}} \otimes \overline{\mathbf{X}}_{j} \overline{\mathbf{X}}_{j}^{H}\right)\left(\mathbf{Z}_{j}^{T} \otimes \mathbf{I}_{N_{t}}\right)^{H}\right] \operatorname{vec}\left(\mathbf{H}^{H}\right)$.

Thus, we have

$$
\sum_{j=1}^{m}\left\|\mathbf{Z}_{j} \mathbf{H} \overline{\mathbf{X}}_{j}\right\|_{F}^{2}=\operatorname{vec}\left(\mathbf{H}^{H}\right)^{H} \mathbf{A} \operatorname{vec}\left(\mathbf{H}^{H}\right),
$$

where $\mathbf{A}=\left(\sum_{j=1}^{m} \mathbf{B}_{j}\right) \in \mathbb{C}^{N_{r} N_{t} \times N_{r} N_{t}}$ so that

$$
\begin{aligned}
\mathbf{B}_{j} & =\left(\mathbf{Z}_{j}^{T} \otimes \mathbf{I}_{N_{t}}\right)\left(\mathbf{I}_{N_{r}} \otimes \overline{\mathbf{X}}_{j} \overline{\mathbf{X}}_{j}^{H}\right)\left(\mathbf{Z}_{j}^{T} \otimes \mathbf{I}_{N_{t}}\right)^{H}, \\
& =\left(\mathbf{Z}_{j}^{H} \mathbf{Z}_{j}\right)^{T} \otimes \overline{\mathbf{X}}_{j} \overline{\mathbf{X}}_{j}^{H} .
\end{aligned}
$$

From [46], the moment generating function of the Hermitian quadratic form in (32) can be written as

$$
\begin{aligned}
\Phi(s) & =\left|\mathbf{I}_{N_{r} N_{t}}-s \mathbf{A}\right|^{-1}, \\
& =\prod_{k=1}^{N_{r} N_{t}}\left(1-s \lambda_{k}(\mathbf{A})\right)^{-1} .
\end{aligned}
$$

Thus, from (27) and (29) we have

$\operatorname{PEP}\left(\mathbf{X}_{i_{1}} \rightarrow \mathbf{X}_{i_{2}} \mid \mathbf{H}\right) \leq \frac{1}{2} \exp \left(-\frac{\rho}{4 M_{t} m n} \sum_{j=1}^{m}\left\|\mathbf{Z}_{j} \mathbf{H} \overline{\mathbf{X}}_{j}\right\|^{2}\right)$

$$
\operatorname{PEP}\left(\mathbf{X}_{i_{1}} \rightarrow \mathbf{X}_{i_{2}}\right) \leq \frac{1}{2} \mathbb{E}\left[\exp \left(-\frac{\rho}{4 M_{t} m n} \sum_{j=1}^{m}\left\|\mathbf{Z}_{j} \mathbf{H} \overline{\mathbf{X}}_{j}\right\|^{2}\right)\right] .
$$

Invoking (36), we have

$$
\operatorname{PEP}\left(\mathbf{X}_{i_{1}} \rightarrow \mathbf{X}_{i_{2}}\right) \leq \frac{1}{2} \prod_{k=1}^{N_{r} N_{t}}\left(1+\frac{\rho}{4 M_{t} m n} \lambda_{k}(\mathbf{A})\right)^{-1} .
$$


If $r=\operatorname{rank}(\mathbf{A})$, then at a high SNR we have

$$
\operatorname{PEP}\left(\mathbf{X}_{i_{1}} \rightarrow \mathbf{X}_{i_{2}}\right) \leq \frac{1}{2}\left(\frac{\rho \lambda_{s m}(\mathbf{A})}{4 M_{t} m n}\right)^{-r}
$$

Thus, the probability of symbol errors at high SNRs can be bounded as

$$
\begin{aligned}
P_{e} & \leq \frac{1}{\left|\mathcal{X}_{i^{*}}\right|} \sum_{\mathbf{X}_{l_{1}} \in \mathcal{X}_{i^{*}}} \sum_{\mathbf{X}_{i_{1}} \neq \mathbf{X}_{i_{2}} \in \mathcal{X}_{i^{*}}} \operatorname{PEP}\left(\mathbf{X}_{i_{1}} \rightarrow \mathbf{X}_{i_{2}}\right), \\
& \leq \frac{\left(\left|\mathcal{X}_{i^{*}}\right|-1\right)}{2}\left(\frac{\rho \lambda_{s m}(\mathbf{A})}{4 M_{t} m n}\right)^{-r} .
\end{aligned}
$$

This concludes the proof.

\section{APPENDIX B}

\section{PROOF OF PROPOSITION 2}

Proof: From Proposition 1 we know that the attainable diversity order of the feedback assisted MIMO system employing (4) is given by $r=\operatorname{rank}\left(\sum_{j=1}^{m} \mathbf{B}_{j}\right)$, where $\mathbf{B}_{j}=$ $\left(\mathbf{Z}_{j}^{H} \mathbf{Z}_{j}\right)^{T} \otimes \overline{\mathbf{X}}_{j} \overline{\mathbf{X}}_{j}^{H}$. We have

$$
\begin{aligned}
\operatorname{rank}\left(\sum_{j=1}^{m} \mathbf{B}_{j}\right) & =\operatorname{rank}\left(\sum_{j=1}^{m}\left(\mathbf{Z}_{j}^{H} \mathbf{Z}_{j}\right)^{T} \otimes \overline{\mathbf{X}}_{j} \overline{\mathbf{X}}_{j}^{H}\right) \\
& \leq N_{r} N_{t}
\end{aligned}
$$

since each matrix $\mathbf{B}_{j}$ is of dimension $N_{r} N_{t} \times N_{r} N_{t}$, the rank of their sum is at most $N_{r} N_{t}$. If the equality holds in (42), it holds for all the values of

$$
\mathbf{B}_{j} \in\left\{\left(\mathbf{Z}_{j}^{H} \mathbf{Z}_{j}\right)^{T} \otimes \overline{\mathbf{X}}_{j} \overline{\mathbf{X}}_{j}^{H} \mid \mathbf{Z}_{j} \in \mathcal{Z}, \overline{\mathbf{X}}_{j} \in \Delta \overline{\mathcal{X}}\right\} .
$$

Thus, the equality should hold for

$$
\begin{aligned}
\mathbf{B}_{j} & \in\left\{\left(\mathbf{Z}_{j}^{H} \mathbf{Z}_{j}\right)^{T} \otimes \overline{\mathbf{X}} \overline{\mathbf{X}}^{H} \mid \mathbf{Z}_{j} \in \mathcal{Z}\right\} \\
& \subset\left\{\left(\mathbf{Z}_{j}^{H} \mathbf{Z}_{j}\right)^{T} \otimes \overline{\mathbf{X}}_{j} \overline{\mathbf{X}}_{j}^{H} \mid \mathbf{Z}_{j} \in \mathcal{Z}, \overline{\mathbf{X}}_{j} \in \Delta \overline{\mathcal{X}}\right\},
\end{aligned}
$$

where $\overline{\mathbf{X}} \in \Delta \overline{\mathcal{X}}$ is a fixed element. In this case, we have

$$
\begin{aligned}
\operatorname{rank}\left(\sum_{j=1}^{m} \mathbf{B}_{j}\right) & =\operatorname{rank}\left(\sum_{j=1}^{m}\left[\left(\mathbf{Z}_{j}^{H} \mathbf{Z}_{j}\right) \otimes\left(\overline{\mathbf{X}} \overline{\mathbf{X}}^{H}\right)^{T}\right]^{T}\right), \\
& =\operatorname{rank}\left(\sum_{j=1}^{m}\left[\left(\mathbf{Z}_{j}^{H} \mathbf{Z}_{j}\right) \otimes\left(\overline{\mathbf{X}} \overline{\mathbf{X}}^{H}\right)^{T}\right]\right) \\
& =\operatorname{rank}\left(\sum_{j=1}^{m}\left[\left(\mathbf{Z}_{j}^{H} \mathbf{Z}_{j}\right)\right] \otimes\left(\overline{\mathbf{X}} \overline{\mathbf{X}}^{H}\right)^{T}\right) \\
& =\operatorname{rank}\left(\sum_{j=1}^{m}\left[\left(\mathbf{Z}_{j}^{H} \mathbf{Z}_{j}\right)\right]\right) \operatorname{rank}\left(\overline{\mathbf{X}} \overline{\mathbf{X}}^{H}\right) \\
& =\operatorname{rank}\left(\overline{\mathbf{Z}}^{H} \overline{\mathbf{Z}}\right) \operatorname{rank}\left(\overline{\mathbf{X}} \overline{\mathbf{X}}^{H}\right) .
\end{aligned}
$$

Thus, it is necessary to have $\operatorname{rank}(\overline{\mathbf{Z}})=N_{r}$ and $N_{t}=\min \{\operatorname{rank}(\overline{\mathbf{X}}) \mid \overline{\mathbf{X}} \in \Delta \overline{\mathcal{X}}\}$ in order to have
$\operatorname{rank}\left(\overline{\mathbf{Z}}^{H} \overline{\mathbf{Z}}\right) \operatorname{rank}\left(\overline{\mathbf{X}} \overline{\mathbf{X}}^{H}\right)=N_{r} N_{t}$ and hence attain the full diversity gain. When the rows of $\mathbf{Z}_{j}$ 's are not orthonormal, we have

$\overline{\mathbf{Z}}=\left[\begin{array}{cccc}\left(\mathbf{Z}_{1} \mathbf{Z}_{1}^{H}\right)^{-1 / 2} & 0 & \cdots & 0 \\ 0 & \left(\mathbf{Z}_{2} \mathbf{Z}_{2}^{H}\right)^{-1 / 2} & \cdots & 0 \\ \vdots & \vdots & \ddots & \vdots \\ 0 & 0 & \cdots & \left(\mathbf{Z}_{m} \mathbf{Z}_{m}^{H}\right)^{-1 / 2}\end{array}\right]\left[\begin{array}{c}\mathbf{Z}_{1} \\ \mathbf{Z}_{2} \\ \vdots \\ \mathbf{Z}_{m}\end{array}\right]$,

and the same necessary condition holds. For sufficiency, consider

$$
\begin{aligned}
\operatorname{rank}\left(\sum_{j=1}^{m} \mathbf{B}_{j}\right) & =\operatorname{rank}\left(\sum_{j=1}^{m}\left[\left(\mathbf{Z}_{j}^{H} \mathbf{Z}_{j}\right) \otimes\left(\overline{\mathbf{X}}_{j} \overline{\mathbf{X}}_{j}^{H}\right)^{T}\right]\right), \\
& =\operatorname{rank}\left(\sum_{j=1}^{m}\left[\left(\mathbf{Z}_{j}^{H} \mathbf{Z}_{j}\right) \otimes\left(\overline{\mathbf{L}}_{j}^{H} \overline{\mathbf{L}}_{j}\right)\right]\right),
\end{aligned}
$$

where $\overline{\mathbf{L}}_{j}$ is the Cholesky factor [47] of $\left(\overline{\mathbf{X}}_{j} \overline{\mathbf{X}}_{j}^{H}\right)^{T}$. Furthermore, we have

$$
\begin{aligned}
\sum_{j=1}^{m}\left[\left(\mathbf{Z}_{j}^{H} \mathbf{Z}_{j}\right) \otimes\left(\overline{\mathbf{L}}_{j}^{H} \overline{\mathbf{L}}_{j}\right)\right] & =\sum_{j=1}^{m}\left[\mathbf{Z}_{j} \otimes \overline{\mathbf{L}}_{j}\right]^{H}\left[\mathbf{Z}_{j} \otimes \overline{\mathbf{L}}_{j}\right] \\
& =\overline{\mathbf{G}}^{H} \overline{\mathbf{G}}
\end{aligned}
$$

where

$$
\overline{\mathbf{G}}=\left[\begin{array}{c}
\mathbf{Z}_{1} \otimes \overline{\mathbf{L}}_{1} \\
\mathbf{Z}_{2} \otimes \overline{\mathbf{L}}_{2} \\
\vdots \\
\mathbf{Z}_{m} \otimes \overline{\mathbf{L}}_{m}
\end{array}\right]
$$

It may be readily shown that if $\operatorname{rank}(\overline{\mathbf{Z}})=N_{r}$, then $\operatorname{rank}(\overline{\mathbf{G}})=N_{r} N_{t}$, since each $\overline{\mathbf{L}}_{j}$ is of rank $N_{t}$ owing to the condition that $\min \{\operatorname{rank}(\overline{\mathbf{X}}) \mid \overline{\mathbf{X}} \in \Delta \overline{\mathcal{X}}\}=N_{t}$. The same condition holds for the case where $\mathbf{Z}_{j}$ 's are not orthonormal. This concludes the proof.

\section{ACKNOWLEDGEMENTS}

The authors acknowledge the use of the IRIDIS High Performance Computing Facility, and associated support services at the University of Southampton, in the completion of this work.

\section{REFERENCES}

[1] I. E. Telatar "Capacity of multi-antenna Gaussian channels," Eur. Trans. Telecommun., vol. 10, no. 6, pp. 585-595, Dec. 1999.

[2] P. Wolniansky, G. Foschini, G. Golden and R. Valenzuela, "V-BLAST: an architecture for realizing very high data rates over the rich-scattering wireless channel," in Proc. International Symp. Signals, Syst., Electron., Pisa, Italy, pp. 295-300, Sep. 1998.

[3] D. Tse and P. Viswanath, "Fundamentals of wireless communication." Cambridge University Press, 2005.

[4] V. Tarokh, N. Seshadri and A. R. Calderbank, "Space-time codes for high data rate wireless communication: performance criterion and code construction," IEEE Trans. Inf. Theory, vol. 44, no. 2, pp. 744-765, Mar 1998.

[5] S. M. Alamouti, "A simple transmit diversity technique for wireless communications," IEEE Journal on Selected Areas in Communications, vol. 16, no. 8, pp. 1451-1458, Oct 1998. 
[6] V. Tarokh, H. Jafarkhani and A. R. Calderbank, "Space-time block codes from orthogonal designs," IEEE Trans. Inf. Theory, vol. 45, no. 5, pp. 1456-1467, Jul 1999.

[7] B. A. Sethuraman, B. S. Rajan and V. Shashidhar, "Full-diversity, highrate space-time block codes from division algebras," IEEE Trans. Inf. Theory, vol. 49, no. 10, pp. 2596-2616, Oct. 2003.

[8] J.-C. Belfiore, G. Rekaya, and E. Viterbo, "The golden code: a $2 \times 2$ full rate space-time code with non-vanishing determinants," IEEE Trans. Inf. Theory, vol. 51, no. 4, pp. 1432-1436, Apr. 2005.

[9] F. Oggier, G. Rekaya, J.-C. Belfiore, and E. Viterbo, "Perfect space-time block codes," IEEE Trans. Inf. Theory, vol. 52, no. 9, pp. 3885-3902, Sept. 2006.

[10] D. J. Love, R. W. Heath and T. Strohmer, "Grassmannian beamforming for multiple-input multiple-output wireless systems," IEEE Trans. Inf. Theory, vol. 49, no. 10, pp. 2735-2747, Oct. 2003

[11] D. J. Love and R. W. Heath, Jr., "Limited feedback unitary precoding for orthogonal space-time block codes," IEEE Trans. Signal Process., vol. 53, no. 1, pp. 64-73, Jan. 2005.

[12] S. Ekbatani and H. Jafarkhani, "Combining beamforming and space-time coding using quantized feedback," IEEE Trans. Wireless Commun., vol. 7, no. 3, pp. 898-908, March 2008

[13] L. P. Natarajan and B. S. Rajan, "Full-rate full-diversity finite feedback space-time schemes with minimum feedback and transmission duration," IEEE Trans. Wireless Commun., vol. 12, no. 10, pp. 5022-5034, Oct. 2013.

[14] R. Mesleh, H. Haas, S. Sinanovic, C. Ahn and S. Yun "Spatial modulation," IEEE Trans. Veh. Technol., vol. 57, no. 4, pp. 2228-2242, July 2008.

[15] M. Di Renzo, H. Haas and P. M. Grant, "Spatial modulation for multipleantenna wireless systems - A Survey," IEEE Commun. Magazine, vol. 49, no. 12, pp. 182-191, Dec. 2011.

[16] A. Stavridis, S. Sinanovic, M. Di Renzo and H. Haas, "Energy evaluation of spatial modulation at a multi-antenna base station," 2013 IEEE 78th Vehicular Technology Conference (VTC Fall), Las Vegas, NV, 2013.

[17] R. Rajashekar, K.V.S. Hari and L. Hanzo, "Spatial modulation aided zero-padded single carrier transmission for dispersive channels," IEEE Trans. Commun., vol. 61, no. 6, pp. 2318-2329, June 2013.

[18] R. Rajashekar, K.V.S. Hari, L. Hanzo, "Reduced-complexity ML detection and capacity-optimized training for spatial modulation systems," IEEE Trans. Commun., vol. 62, no. 1, pp. 112-125, Jan. 2014.

[19] P. Yang, M. Di Renzo, Y. Xiao, S. Li and L. Hanzo, "Design guidelines for spatial modulation," IEEE Communications Surveys \& Tutorials, vol. 17, no. 1, pp. 6-26, First quarter 2015.

[20] E. Basar, U. Aygolu, E. Panayirci and H. V. Poor, "Space-time block coding for spatial modulation," IEEE Trans. Commun., vol. 59, no. 3, pp. 823-832, Mar. 2011.

[21] R. Rajashekar and K.V.S. Hari, "Modulation diversity for spatial modulation using complex interleaved orthogonal design," in Proc. IEEE TENCON 2012, Nov. 2012, pp. 1-6.

[22] X. Li and L. Wang, "High rate space-time block coded spatial modulation with cyclic structure," IEEE Commun. Lett., vol. 18, no. 4, pp. 532-535, Apr. 2014

[23] A. G. Helmy, M. Di Renzo and N. Al-Dhahir, "Enhanced-reliability cyclic generalized spatial-and-temporal modulation," IEEE Commun Lett., vol. 20, no. 12, pp. 2374-2377, Dec. 2016.

[24] P. Yang, Y. Xiao, L. Li, Q. Tang, Y. Yu and S. Li, "Link adaptation for spatial modulation with limited feedback," IEEE Trans. Veh. Technol., vol. 61, no. 8, pp. 3808-3813, Oct. 2012.

[25] R. Rajashekar, K.V.S. Hari and L. Hanzo, "Antenna selection in spatial modulation systems," IEEE Commun. Lett., vol. 17, no. 3, pp. 521-524, Mar. 2013.

[26] Z. Zhou, N. Ge and X. Lin, "Reduced-complexity antenna selection schemes in spatial modulation," IEEE Commun. Lett., vol. 18, no. 1, pp. 14-17, Jan. 2014.

[27] N. Wang, W. Liu, H. Men, M. Jin, and H. Xu, "Further complexity reduction using rotational symmetry for EDAS in spatial modulation," IEEE Commun. Lett., vol. 18, no. 10, pp. 1835-1838, Oct. 2014.

[28] R. Rajashekar, K.V.S. Hari and L. Hanzo, "Quantifying the transmit diversity order of Euclidean distance based antenna selection in spatial modulation," IEEE Signal Proc. Lett., vol. 22, no. 9, pp. 1434-1437, Sept. 2015.

[29] Z. Sun, Y. Xiao, L. You, L. Yin, P. Yang and S. Li, “Cross-entropy-based antenna selection for spatial modulation," IEEE Commun. Lett., vol. 20, no. 3, pp. 622-625, March 2016.

[30] P. Yang, Y. Xiao, Y. L. Guan, S. Li and L. Hanzo, "Transmit antenna selection for multiple-input multiple-output spatial modulation systems," IEEE Trans. Commun., vol. 64, no. 5, pp. 2035-2048, May 2016.
[31] Z. Sun et. al., "Transmit antenna selection schemes for spatial modulation systems: search complexity reduction and large-scale MIMO applications," to appear in IEEE Trans. Veh. Technol..

[32] D. A. Gore and A. J. Paulraj, "MIMO antenna subset selection with space-time coding," IEEE Trans. Signal Process., vol. 50, no. 10, pp. 2580-2588, Oct. 2002.

[33] A. Gorokhov, D. A. Gore and A. J. Paulraj, "Receive antenna selection for MIMO spatial multiplexing: theory and algorithms," IEEE Trans. Signal Process., vol. 51, no. 11, pp. 2796-2807, Nov. 2003.

[34] A. F. Coskun and O. Kucur, "Performance analysis of joint single transmit and receive antenna selection in Nakagami-m fading channels," IEEE Commun. Lett., vol. 15, no. 2, pp. 211-213, Feb. 2011

[35] M. Jain et. al., "Practical, real-time, full duplex wireless." MobiCom '11, pages 301-312, New York, NY, USA, 2011. ACM.

[36] M. Duarte, C. Dick, and A. Sabharwal, "Experiment-driven characterization of full-duplex wireless systems," IEEE Trans. Wireless Commun., vol. 11, no. 12, pp. 4296-4307, Dec. 2012

[37] D. Bharadia and S. Katti, "Full duplex mimo radios" in Proc. 11th USENIX Symp. NSDI, Seattle, WA, USA, pp. 359-372, Apr. 2014.

[38] B. Jiao, M. Wen, M. Ma and H. V. Poor, "Spatial modulated full duplex," IEEE Wireless Commun. Lett., vol. 3, no. 6, pp. 641-644, Dec. 2014.

[39] P. Raviteja, Y. Hong and E. Viterbo, "Spatial modulation in full-duplex relaying," IEEE Commun. Lett., vol. 20, no. 10, pp. 2111-2114, Oct. 2016.

[40] J. Zhang, Q. Li, K. J. Kim, Y. Wang, X. Ge and J. Zhang, "On the performance of full-duplex two-way relay channels with spatial modulation," IEEE Trans. Commun., vol. 64, no. 12, pp. 4966-4982, Dec. 2016.

[41] F. Jiang and A. L. Swindlehurst, "Optimization of UAV heading for the ground-to-air uplink," IEEE Journal on Selected Areas in Communications, vol. 30, no. 5, pp. 993-1005, June 2012.

[42] Y. Zeng, R. Zhang and T. J. Lim, "Wireless communications with unmanned aerial vehicles: opportunities and challenges," IEEE Communications Magazine, vol. 54, no. 5, pp. 36-42, May 2016.

[43] F. Ono, H. Ochiai, K. Takizawa, M. Suzuki, and R. Miura, "Performance analysis of wireless relay network using network coding and UAS," in Proc. Globecom Workshops (GC Wkshps), Atlanta, GA, USA, pp. 14091414, Dec. 2013

[44] W. Guo, C. Devine, and S. Wang, "Performance analysis of micro unmanned airborne communication relays for cellular networks," in Proc. IEEE/IET Int. Symp. Commun. Syst. Netw. Digit. Signal Process. (CSNDSP), Manchester, U.K., pp. 658-663, Jul. 2014

[45] S. Chandrasekharan et. al., "Designing and implementing future aerial communication networks," IEEE Communications Magazine, vol. 54, no. 5, pp. 26-34, May 2016.

[46] G. L. Turin, "The characteristic function of Hermitian quadratic forms in complex normal random variables," Biometrika, vol. 47, no. 1/2, pp. 199-201, Jun. 1960.

[47] G. H. Golub, "Matrix computations," Johns Hopkins University, 1983.

[48] A. W. Ingleton, "The rank of circulant matrices" J. London Math. Soc. s1-31 (4): 445-460. doi:10.1112/jlms/s1-31.4.445

[49] N. J. A. Sloane, "Triangle of coefficients of cyclotomic polynomial," A013595 - The on-line encyclopedia of integer sequences (OESI).

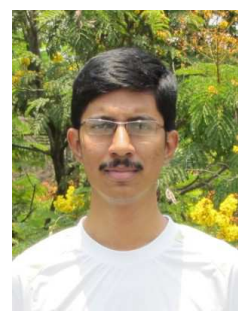

Rakshith Rajashekar (M'14) received the B.E. degree in electrical communication engineering from Visvesvaraya Technological University, Karnataka, India, in 2007. He received his Ph.D.from the Department of Electrical Communication Engineering, Indian Institute of Science (IISc), India, in 2014 $\mathrm{He}$ is presently working as a Research Fellow at the University of Southampton (UoS), UK. Before joining the UoS, he worked at Accord Software \& Systems, Bangalore, India, as a Systems Engineer from 2007 to 2009, and as a Senior Scientist at Broadcom Communications, Bangalore, India from 2014 to 2015. His research interests include antenna selection in MIMO systems, differential communication, millimeter wave communication with a focus on space-time signal processing and coding. 


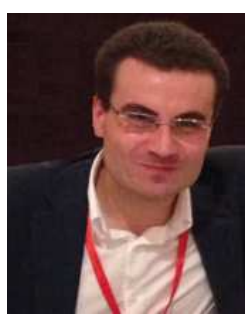

Marco Di Renzo (S'05-AM'07-M'09-SM'14) received the Laurea (cum laude) and the Ph.D. degrees in electrical engineering from the University of L'Aquila, Italy, in 2003 and in 2007, respectively, and the Doctor of Science degree (HDR) from the University Paris-Sud, France, in 2013. Since 2010, he has been a CNRS Associate Professor ("Chargé de Recherche Titulaire CNRS") in the Laboratory of Signals and Systems of Paris-Saclay University - CNRS, CentraleSupélec, Univ Paris Sud, Paris, France. $\mathrm{He}$ is an Adjunct Professor at the University of Technology Sydney, Australia, a Visiting Professor at the University of L'Aquila, Italy, and a co-founder of the university spin-off company WEST Aquila s.r.l., Italy. He serves as the Associate Editor-in-Chief of IEEE COMMUNICATIONS LETTERS, and as an Editor of IEEE TRANSACTIONS ON COMMUNICATIONS, and IEEE TRANSACTIONS ON WIRELESS COMMUNICATIONS. He is a Distinguished Lecturer of the IEEE Vehicular Technology Society and IEEE Communications Society. He is a recipient of several awards, including the 2013 IEEE-COMSOC Best Young Researcher Award for Europe, Middle East and Africa (EMEA Region), the 2014-2015 Royal Academy of Engineering Distinguished Visiting Fellowship, the 2015 IEEE Jack Neubauer Memorial Best System Paper Award, and the 2015-2018 CNRS Award for Excellence in Research and in Advising Doctoral Students.

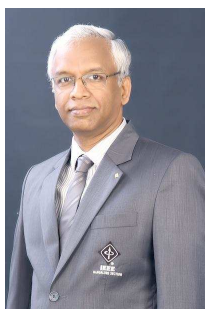

K.V.S. Hari (M'92-SM'97-F'15) received the B.E. degree from Osmania University, Hyderabad, India, in 1983; the M.Tech. degree from the Indian Institute of Technology Delhi, New Delhi, India, in 1985; and the Ph.D. degree from the University of California at San Diego, La Jolla, CA, USA, in 1990.Since 1992, he has been with the Department of Electrical Communication Engineering, Indian Institute of Science, Bangalore, India, where he is currently a Professor and coordinates the activities of the Statistical Signal Processing Laboratory. He was an Affiliated Professor (2010-16) with the Department of Signal Processing, KTH Royal Institute of Technology, Stockholm, Sweden. He has been a Visiting Faculty Member with Stanford University, Stanford, CA, USA; KTH Royal Institute of Technology, Stockholm, Sweden; and Aalto University, Espoo, Finland (formerly Helsinki University of Technology). While at Stanford University, he worked on multiple-input multiple-output (MIMO) wireless channel modelling and co-authored the Worldwide Interoperability for Microwave Access standard on wireless channel models for fixed-broadband wireless communication systems, which proposed the Stanford University Interim channel models. He was also with the Defence Electronics Research Laboratory, Hyderabad, and the Research and Training Unit for Navigational Electronics, Osmania University. His research interests include the development of signal processing algorithms for MIMO wireless communication systems, sparse signal recovery problems, indoor positioning, assistive technologies for the elderly, and neuroscience. Dr. Hari was cu an Editor of Elsevier's EURASIP journal Signal Processing (2006-16) and the Senior Associate Editor of Springer's Indian Academy of Sciences journal SADHANA. He is an Academic Entrepreneur and a Cofounder of the company ESQUBE Communication Solutions, Bangalore. He received the Institution of Electronics and Telecommunication Engineers S. V. C. Aiya Award for Excellence in Telecom Education and the Distinguished Alumnus Award from the Osmania University College of Engineering, Hyderabad. He is a Fellow of the Indian NAE.

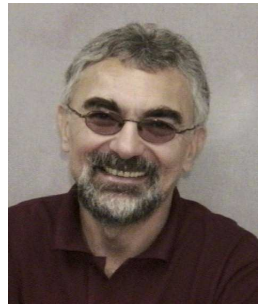

Lajos Hanzo (http://www-mobile.ecs.soton.ac.uk) FREng, FIEEE, FIET, Fellow of EURASIP, DSc received his degree in electronics in 1976 and his doctorate in 1983. In 2009 he was awarded an honorary doctorate by the Technical University of Budapest and in 2015 by the University of Edinburgh. In 2016 he was admitted to the Hungarian Academy of Science. During his 40-year career in telecommunications he has held various research and academic posts in Hungary, Germany and the UK. Since 1986 he has been with the School of Electronics and Computer Science, University of Southampton, UK, where he holds the chair in telecommunications. He has successfully supervised 111 $\mathrm{PhD}$ students, co-authored 18 John Wiley/IEEE Press books on mobile radio communications totalling in excess of 10000 pages, published 1685 research contributions at IEEE Xplore, acted both as TPC and General Chair of IEEE conferences, presented keynote lectures and has been awarded a number of distinctions. Currently he is directing a 60 -strong academic research team, working on a range of research projects in the field of wireless multimedia communications sponsored by industry, the Engineering and Physical Sciences Research Council (EPSRC) UK, the European Research Council's Advanced Fellow Grant and the Royal Society's Wolfson Research Merit Award. He is an enthusiastic supporter of industrial and academic liaison and he offers a range of industrial courses. He is also a Governor of the IEEE VTS. During 2008 - 2012 he was the Editor-in-Chief of the IEEE Press and a Chaired Professor also at Tsinghua University, Beijing. For further information on research in progress and associated publications please refer to http://wwwmobile.ecs.soton.ac.uk Lajos has $30000+$ citations and an H-index of 68. 